This document is the Accepted Manuscript version of a Published Work that appeared in final form in Biomacromolecules, copyright (c) 2019, American Chemical Society after peer review and technical editing by the publisher. To access the final edited and published work see https://doi.org/10.1021/acs.biomac.8b01382

\title{
3D Scaffolds based on Conductive Polymers for
}

\section{Biomedical Applications}

\author{
Nuria Alegret, ${ }^{a, b}$ Antonio Dominguez-Alfaro, ${ }^{a, c}$ and David Mecerreyes. ${ }^{a, d}$ \\ ${ }^{a}$ POLYMAT University of the Basque Country UPV/EHU, Avenida de Tolosa 72, 20018 \\ Donostia-San Sebastián, Spain \\ ${ }^{\mathrm{b}}$ Cardiovascular Institute, School of Medicine, Division of Cardiology, University of Colorado \\ Denver Anschutz Medical Campus, 12700 E.19th Avenue, Bldg. P15, Aurora, CO, 80045, USA. \\ ${ }^{\mathrm{c}}$ Carbon Nanobiotechnology Group, CIC biomaGUNE, Paseo de Miramón 182, 2014 \\ Donostia-San Sebastián, Spain \\ d Ikerasque, Basque Foundation for Science, 48013 Bilbao, Spain.
}

KEYWORDS. Conjugated Polymers, 3D scaffold, polypyrrole, PEDOT, PANi, biomedical applications, tissue engineering, drug delivery, electric stimulation.

ABSTRACT. 3D scaffolds appear to be a cost-effective ultimate answer for biomedical applications, facilitating rapid results while providing an environment similar to in vivo tissue with large surface areas for cell or biomaterial attachment, proliferation, and sensing. In addition to regenerative applications, a larger surface area can be an asset for biosensing and drug delivery applications, providing increased sensitivity and concentration range and higher drug loads. 
Conductive 3D scaffolds are composed of conjugated polymers (CPs) and natural non-conductive polymers arranged in a 3D architecture which provides tridimensionality to cellular culture, and thus a closer-to-in vivo environment, along with a high surface area for cell adherence and proliferation. However, the scaffolds must also obey other characteristics: homogeneous porosity, with pore sizes large enough to allow cell penetration and nutrient flow; elasticity and wettability similar to the tissue of implantation; and a suitable composition to enhance cell-matrix interactions. In this review we summarize the fabrication methods, characterization techniques and main applications of conductive 3D scaffolds based on conductive polymers. The main barrier in the development of these platforms has been the fabrication and subsequent maintenance of the third dimension due to challenges in the manipulation of conductive polymers. In the last decades, different approaches to overcome these barriers have been developed for the production of conductive 3D scaffolds, demonstrating a huge potential for biomedical purposes. Finally, we present an overview of the emerging strategies developed to manufacture 3D conductive scaffolds, the techniques used to fully characterize them, and the biomedical fields where they have been applied. 


\section{The importance of using conductive scaffolds in biomedical sciences}

Many functions of the human body are regulated by electric signals, including neural communication, embryonic development, tissue repair after injury, and heartbeat. ${ }^{1}$ The nervous system is the largest electrically active infrastructure in our body, followed by the heart and other muscles. $^{2-3}$ The former uses electricity to transmit information from neuron to neuron through synapses while the muscle cells of the heart produce electrical impulses that travel through the entire organ leading to muscle contraction, thus producing rhythmical heart beats. In addition, several studies have also demonstrated that some tissues, such as bone marrow, use conductivity to regenerate new tissue. ${ }^{4}$ Therefore, materials that interface with electrically active tissues for bioapplication purposes such as tissue engineering or monitoring, must also be conductive to enhance the biological response to external stimuli.

Conjugated polymers (CPs) are a composite of an oxidized conjugated polymer backbone and a negatively charged species (known as a dopant) that counterbalances the overall charge. The key feature that makes a CP highly conductive is the oxidized state that, in combination with the conjugated backbone and the dopant, allows the free and rapid electronic movement along the polymeric structure. CPs are an optimal choice for the design of devices and scaffolds with electric properties, not only to provide conductivity, but also because of their high biocompatibility. ${ }^{5}$ Polypyrrole (PPy), polyaniline (PANI) and poly(3,4-ethyl-enedioxythiophene) (PEDOT) have been by far the most utilized CPs in biomedical fields, and have been the only ones used in the development of conductive 3D architectures for bioapplications. As illustrated in Figure 1, conductive polymer 3D Scaffolds have been applied mainly as three different architectures: porous scaffolds, fiber meshes and hydrogels. ${ }^{6}$ 

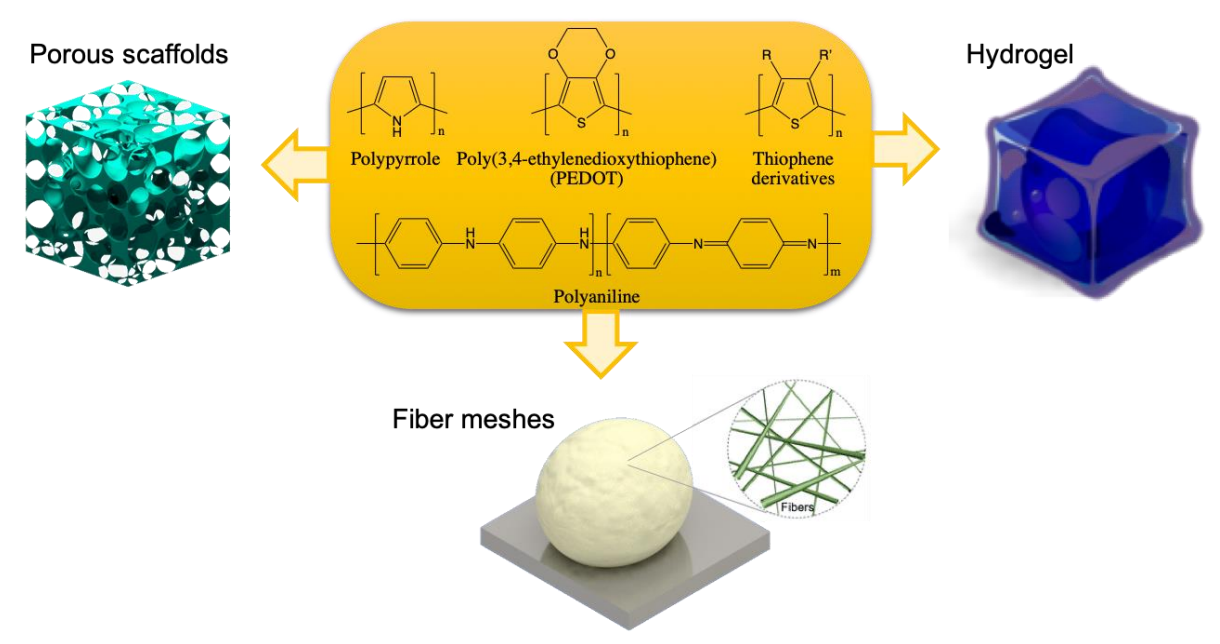

Figure 1. Conductive 3D architectures manufactured from conjugated polymers.

\subsection{Importance of $3 D$ cell cultures}

To ensure complete acceptance by the host tissue, the implants must mimic as much as possible the biological environment porosity, permeability and mechanical stability. Current in vitro tests to evaluate material suitability and toxicity rely on bidimensional cell cultures, and do not fully embrace the complexity and heterogeneity of real biological systems. One alternative might be animal models; however, their high cost, time-consuming screenings, and ethical issues make them unfeasible for most projects and reserve them for definitive tests and evaluations. Thus, 3D scaffolds appear as an eest-effective ultimate answer for biomedical applications obtaining rapid results while providing an environment similar to in vivo tissues with large surface areas for cell or biomaterial attachment, proliferation, sensing, etc. ${ }^{7}$ Several studies have demonstrated that cells have a behavior and response when cultured in a 3D organization that is closer to the in vivo, providing a more realistic predictive outcome. ${ }^{8}$

The proper scaffold must comply with the following characteristics: (i) tridimensional porous structure, with pore size large enough to allow cellular penetration and growth, nutrient and metabolic waste flow; (ii) mechanical properties similar to the tissue it is to be placed in; and (iii) 
a suitable surface composition and morphology permitting cellular adhesion and interaction. ${ }^{9}$ This third feature is the dominant factor for a successful adaptability of the scaffold inside the biological environment. To modulate and enhance such interactions, a large variety of biocompatible polymers, dopants, compositions and biomaterials have been used in the design of CPs 3D scaffolds. Most of them are porous materials, hydrogels and fiber meshes, composed of nonconductive natural polymers mixed with CPs permitting acquisition of electrical properties. ${ }^{7}$ The most significant ones are reviewed along these pages.

\subsubsection{What to consider $3 D$ vs $2 D$ scaffolds}

The tridimensional feature has been examined in different ways. Due to the recent novelty of this concept in biomedicine, there is not a clear distinction between 2D and 3D dimensionality. Some authors considered 3D structures as tridimensional when their thickness exceeds few nanometers in all three directions, even though the z-axis is only in the order of hundreds of nanometers, such as fibers membranes. ${ }^{10}$ Other authors restrict 3D substrates to those measuring a few microns in height, ${ }^{11}$ while some were able to construct scaffolds of a few millimeters. Additionally, the term "3D" has been applied to material-cell assemblies. ${ }^{12}$

Furthermore, pore size is a feature to consider when defining tridimensionality. For instance, traditional fibrous meshes, even those of mm thickness, can have pore sizes up to few microns, thus providing superficial porosity but hindering cell infiltration inside the structure, thus hampering achieving a 3D culture. ${ }^{13-14}$ Pore sizes of $200-500 \mu \mathrm{m}$ have been suggested as the most effective size to favor tissue growth. ${ }^{15}$

Herein, we have defined our own limits of tridimensionality that must apply for any scaffold to be considered 3D: the length in each direction must be a minimum of double the average height of the studied cell. For example, if cardiomyocytes, with known thickness of around $20 \mu \mathrm{m},{ }^{16}$ are 
incubated, the 3D scaffold must have a height of at least $40 \mu \mathrm{m}$. Alternatively, if culturing neurons with a thickness of $150-200 \mu \mathrm{m},{ }^{17}$ then the substrate must have a thickness of at least $300 \mu \mathrm{m}$. Furthermore, few publications specify a conductive 3D scaffold under our definition. We believe that these characteristics (dimensions of the 3D, size of the porosity and conductivity), are required to obtain the best predictive result in biomedical applications. Therefore, this is an up to date overview of the different processing and characterization methods used to manufacture 3D scaffolds as well as their most relevant applications. All the works presented here fulfill our 3D definition.

\section{Fabrication methods of 3D scaffolds based on conducting polymers}

\subsection{Porous scaffolds}

The main drawbacks of using CPs in biomedical devices are the poor mechanical properties and their difficulties in manipulating and processing. They are commonly stiff, brittle and insoluble, leading to delamination and poor durability. Blending CPs with other malleable non-conductive polymers has become a convenient solution to overcome such shortcomings, combining the

positive qualities of both materials. ${ }^{6,18}$ Although the preparation of 3D CP-based materials is tricky, in recent years the number of manufacturers of such scaffolds has increased significantly. A diverse collection of processing methods has been used, and all of them have in common the use of a template to define the $3 \mathrm{D}$ geometrical shape as well as the internal configuration. A summary is shown in Figure 2 and a detailed discussion of each process will be presented in this section. 


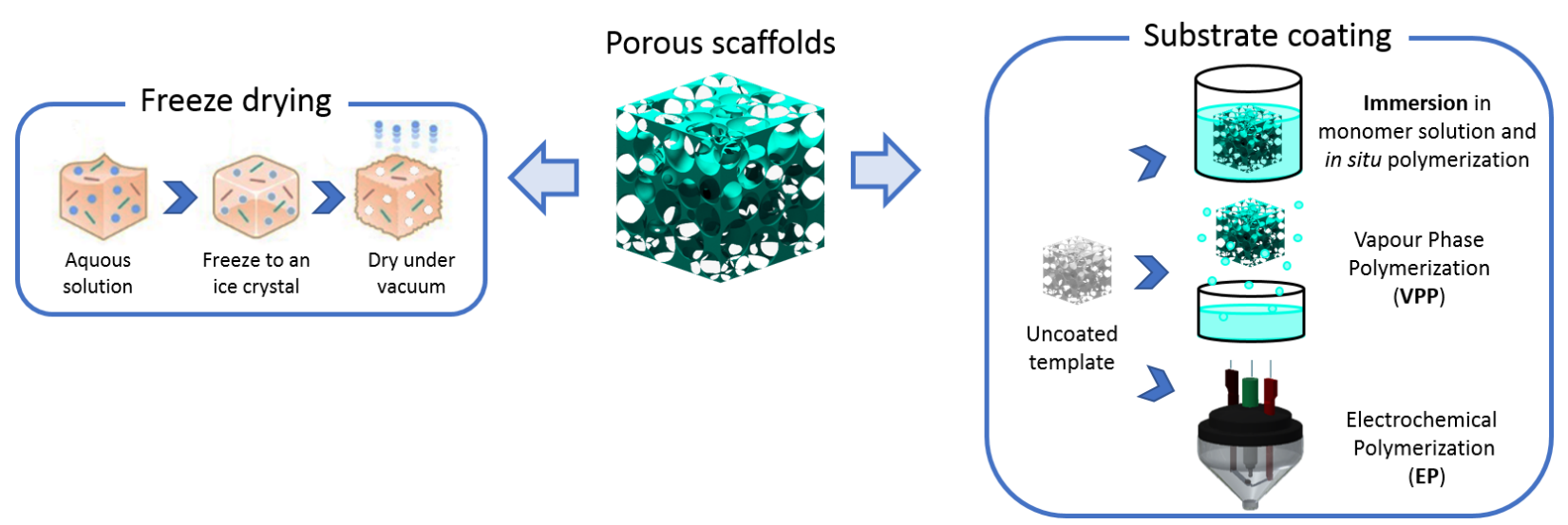

Figure 2. Processing methods for manufacturing porous scaffolds.

The most conventional method used is polymerization in solution of the monomer in the presence of an oxidant and other non-conductive polymers. ${ }^{19}$ Although conductive hydrogels have been synthesized since 1994 (see next section), ${ }^{20}$ the first attempt in manufacturing a 3D porous conductive scaffold appeared in 2013, when Sajesh and co-workers used alginate (Alg), a natural biopolymer that allows cell penetration and encapsulation, to functionalize polypyrrole (PPy) and become incorporated into a chitosan matrix. PPy was first polymerized via chemical oxidation with $\mathrm{FeCl}_{3}$ of a pyrrole solution containing Alg. The blend was next added to a chitosan solution, which was then lyophilized and crosslinked with glutaraldehyde. ${ }^{21}$

Freeze-drying or lyophilization uses ice or frozen solvent as a template to build the tridimensional structure. First, a mixed polymer solution containing confined solute particles within the solvent crystals is poured into the desired mold and frozen. Second, through ice sublimation, a cast 3D network is formed retaining the solute particles in their original position and conformation. ${ }^{22-24}$ Freeze-drying is a versatile and facile method to produce 3D macroporous structures with any shape and dimension imaginable. ${ }^{25}$ Several studies have demonstrated that 
variation of the parameters, such as composition, freezing and/or sublimation rate and temperatures, can influence the condition of the final substrate, as well as modulate its porosity. ${ }^{25}$ The most common method to incorporate CPs is by in situ polymerization and deposition into an already prepared 3D substrate, also known as 'coating'. ${ }^{26-27}$ Vapour phase polymerization (VPP) has become a feasible method to deposit conductive films in a defined and controllable manner. In contrast to conventional chemical polymerization in solution, synthesis of the polymer in the VPP process occurs through a monomer in its vapour state. Usually, the process takes place in three steps. First, the prepared substrate is soaked into an oxidant solution. Second, it is placed inside a closed chamber or container together with a monomer solution. It has to be noted that both species inside the chamber must not be in direct contact. Third, the coated substrate is rinsed in an alcohol solvent, such as butanol and water, to remove any unreacted oxidant and monomer. The monomer vapour can be formed through several procedures, and heat or vacuum under an inert atmosphere are the most common methods to evaporate the monomer. ${ }^{28}{ }^{29}$ Even a combination of both heat and vacumm can be a proper choice for those monomers with a high boiling point, such as EDOT $\left(193^{\circ} \mathrm{C}\right)$. As an alternative, the monomer vapour can be stored or produced in a separated connected chamber, while the vapour flow is guided through pressure differences between them. ${ }^{30}$ The chemical synthesis takes place by the polymerization of the monomer vapour inside the 3D substrate. In order to slow down the PEDOT polymerization process, Iandolo et al. added pyridine or the triblock copolymer PEG-PPG-PEG into the oxidant solution to reduce the oxidant reactivity, since it had been previously shown to avoid the crystallite formation of iron tosylate and favour the PEDOT guiding during its synthesis. ${ }^{29}$

In other cases, the approach to fabricate macro-porous $3 \mathrm{D}$ substrates includes the use of a template, which can be removed at the end of the process, resulting in the $3 \mathrm{D}$ replica structure. 
Polystirene (PS) and poly(lactic-co-glycolic acid) (PLGA) are the polymers used for this role. ${ }^{7,31}$ Afterwards, both template microspheres can be selectively removed by the addition DCM.

Alternatively, electrochemical deposition may be used for coating 3D substrates. Song et al. developed a simple and low-cost method using nickel foam as a template to manufacture foamlike scaffolds composed of reduced-graphene oxide (rGO) and PPy. ${ }^{32}$ They combined an electrostatic layer-by-layer (LBL) assembly methodology to first absorb the rGO nanosheets within the template with an electrochemical deposition strategy, and then polymerized PPy on the rGO-based substrate. At the end, the Ni was eliminated via etching, resulting in a highly porous and flexible scaffold. In another example, a bulk sponge made of carbon nanotubes (CNTs) has been directly used as working electrode to electrodeposite PANI using a three-electrode cell. ${ }^{33}$

Severt and co-workers went a step further in the coating methodology and combined both chemical and electrochemical polymerization to sequencially deposit CP onto sponge-like silk substrates. ${ }^{18}$ After first depositing a layer of PPy via chemical polymerization, the resulting porous scaffolds were conductive enough to serve as working electrodes for a posterior electropolymerization of either PPy, PEDOT or PEDOT-OH, thus obtaining an additional layer of $\mathrm{CP}$ on the surface of the scaffold. The authors observed that such an additional layer reduces the resistivity of the substrate and increases its stability during long-term storage.

The most simple and final processing method reported does not include a polymerization step, but utilizes already available commercial polymer solutions, such as the commonly used PEDOT:PSS Clevios PH-1000, from Heraeus. Ding and co-workers covered a melamine sponge with a PEDOT:PSS by simply dipping the sponge into a polymeric solution and then letting it dry in a vaccumm oven. ${ }^{34}$ Even though this is the fastest and easy way used to generate conductive 3D scaffolds ever published, delamination easily occurs due to the weak interaction between the CP 
and the substrate. Thus, the commercial CP solutions are usually mixed with other non-conjugated polymers, placed in molds and freeze-dried. ${ }^{25}$ Most protocols use gelatin, chitosan or collagen to later crosslink and improve the mechanical properties and stability of the final porous scaffolds. $23,35-37$

\subsection{Hydrogels based on conductive polymers}

During the past decades, hydrogels have received special attention due to their unique and inherent properties. Their highly hydrated nature, together with their flexibility and softness, make them outstanding biomimetic materials for soft tissue applications. ${ }^{38-40}$ Furthermore, conductive hydrogels may also afford electrical properties permitting their use as bioelectrodes in applications that require interfacing with soft tissues. ${ }^{41-42}$ Similar to the conductive porous scaffolds in the previous section, such electrical properties can be incorporated by addition of CPs through polymerization before, after or even at the same time as the gel crosslinking (Figure 3 ). ${ }^{43}$

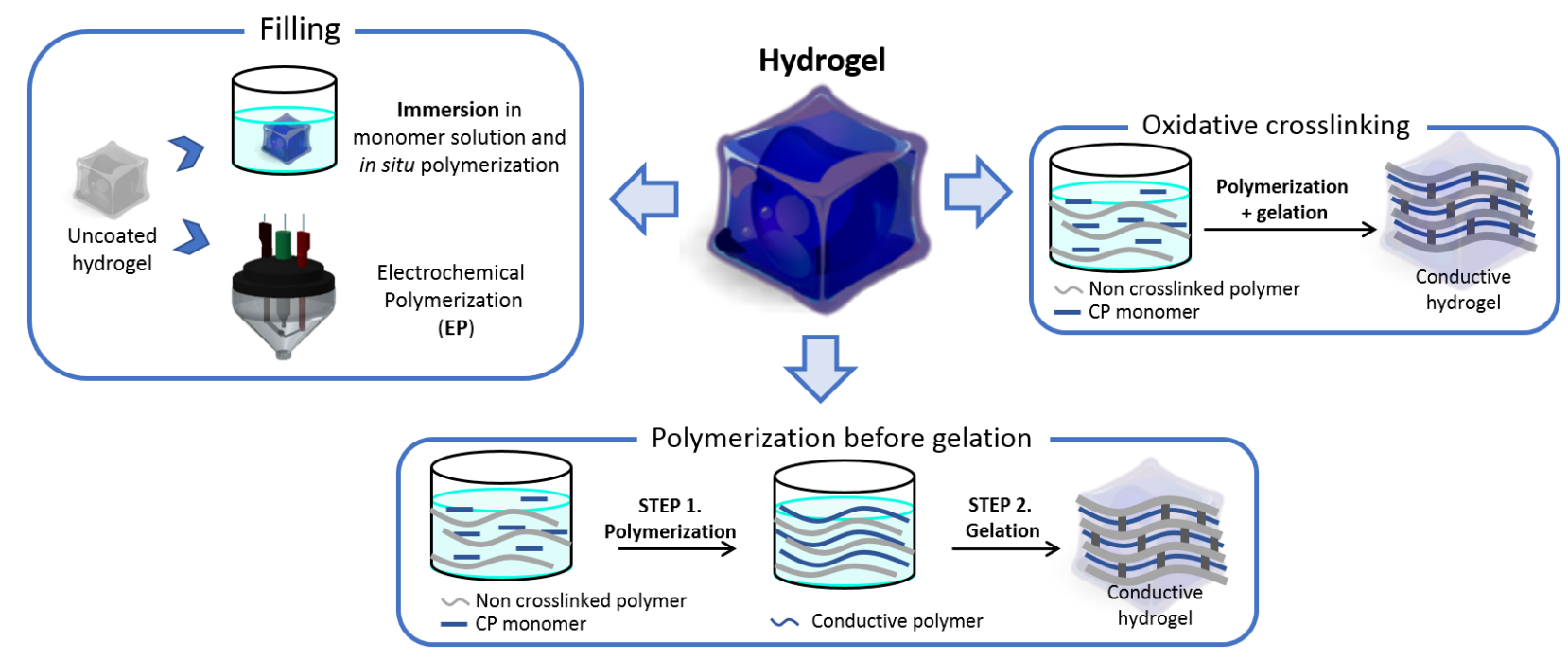

Figure 3. Processing methods for manufacturing conductive hydrogels. 
The processing methodologies employed so far can be considered easy and feasible, using in most cases natural biopolymers, such as chitosan or gelatin, as backbones for the hydrogels. ${ }^{44}$ Yang et al. used a simple chemical polymerization of PPy within an already crosslinked alginate (Alg) hydrogel, a natural polysaccharide that forms ionic crosslinked networks in presence of divalent cations. ${ }^{43}$ In their work, the authors introduced the hydrogel in an aqueous pyrrole solution, allowing the monomer to penetrate within the gel by diffusion. Then, the polymerization was developed by adding the $\mathrm{FeCl}_{3}$ oxidant inside the solution, resulting in highly homogeneous PPy/Alg hydrogels. In other reports, the incorporation order of the reagents within the hydrogel has been reversed. $\mathrm{Xu}$ and co-workers immersed crosslinked carboxymethyl chitosan (CMCS) hydrogels into an oxidant solution of ammonium persulphate (APS), and then into EDOT/hexane solution to perform the subsequent polymerization. In this case, the authors applied vacuum to ensure a uniform dispersion of the oxidant within the gel structure, and a vibrator to facilitate the polymerization process. ${ }^{45}$

Electrochemical polymerization within a gel has also been achieved by Mario Cheong and coworkers. ${ }^{46}$ They first prepared a sericin and PVA hydrogel coated on a glass substrate with a prelayer of PEDOT/pTS. This both enhances adherence of hydrogels to substrates and introduces conductivity for use as an electrode. Later, the hybrid was dipped into an aqueous EDOT solution and subsequently PEDOT was polymerized by electrodeposition at low charge density, achieving a slow uniform deposition throughout the substrate. According to the authors, such homogeneity is essential to avoid increased voltage across the electrode, which would damage the hydrogel through hydrolysis, and lead to the formation of a nodular polymer with high peaks that can breach the hydrogel surface. 
A second process to introduce CPs into a hydrogel performs the polymerization of the CP and the crosslinking at the same time. In this case, two solutions must be prepared, one containing the biopolymer and the $\mathrm{CP}$ monomer, and the other the oxidant and the crosslinker. Both the polymerization and gelation start as soon as the solutions are mixed. ${ }^{47}$ Shin and co-workers developed an oxidative crosslinking using sodium periodate $\left(\mathrm{NaIO}_{4}\right)$ as a double agent, acting both as oxidant for EDOT monomer and crosslinker for catechol-functionalized hyaluronic acid (HACA) ${ }^{48}$ Furthermore, other substances can be added to stabilize the final structure or modulate its final properties.

Finally, the last methodology includes using a solution of already synthesized or commercial CP as a starting point, adding the hydrogel precursor and, afterwards, the crosslinker to form the final gel. ${ }^{49}$ Mawad et al. prepared a N-(3-aminopropyl) methacrylamide hydrochloride (APMA) derived-PEDOT crosslinked with acrylic acid (AA) in presence of poly(ethylene glycol) diacrylate $(\mathrm{PEG}-\mathrm{DA}) .{ }^{50}$

Single component conductive hydrogels have also been achieved. In the study performed by Dai et al., hydrogels composed of PEDOT/PSS where manufactured through a type of ionic crosslinking using an excess of $\mathrm{Fe}^{3+}$ ions. ${ }^{51}$ As well, monomer derivatives have been synthesized to achieve gelation not only through crosslinking of the side chains, ${ }^{52}$ but also without such cross/linking effect: Du and co-workers succeeded in the formation of PEDOT-based hydrogels from an amphiphilic PEDOT monomer, yielding to a non-crosslinked 3D structure as shown in Figure 4). ${ }^{53}$ 


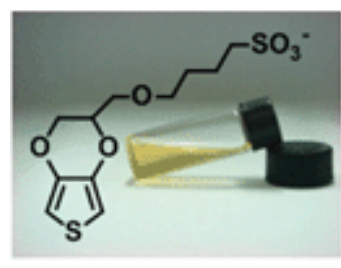

\section{Polymerization}

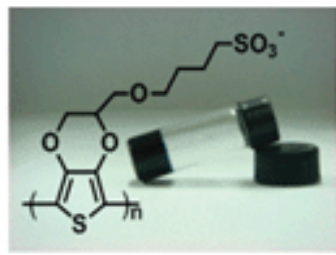

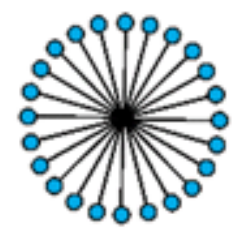

EDOT-S colloid
APS, or $\mathrm{FeCl}_{3}$, or mixture of both

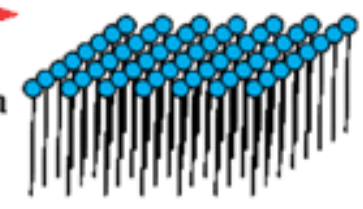

\section{PEDOT-S hydrogel}

Figure 4. Schematic diagram of the synthesis of conducting polymer hydrogels: An EDOT-S colloidal solution with spherical micelles has converted into a PEDOT-S hydrogel with sheet-like building blocks upon addition of an oxidant. Reprinted with permission from ref. 53 Copyright (2011) Royal Society of Chemistry.

\subsection{Electrospun fiber meshes}

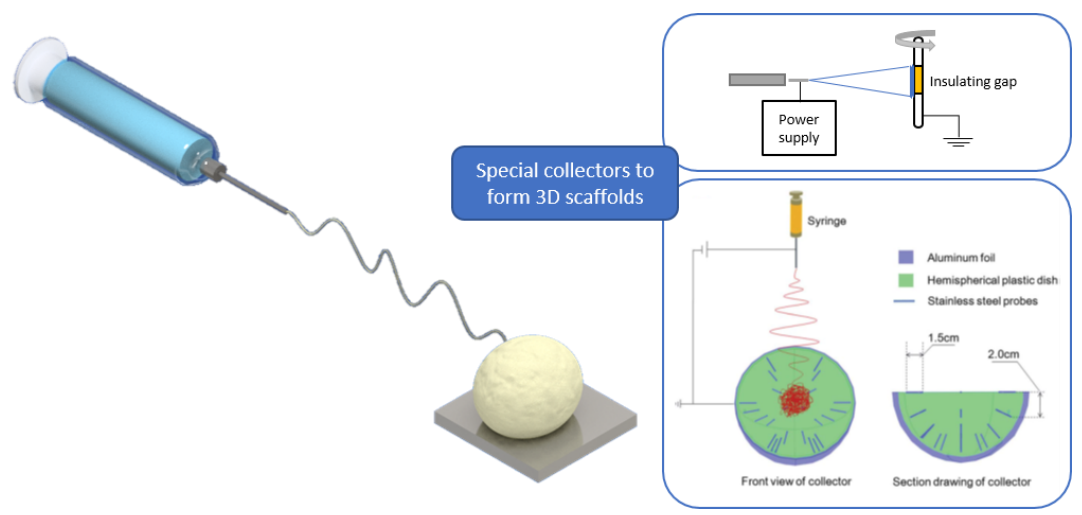

Figure 5. Schematic representation of the fabrication of $3 \mathrm{D}$ electrospun fibrous scaffolds. Adapted with permission from ref 14. Copyright (2012) Royal Society of Chemistry.

Fibers are the third type of 3D scaffolds used in tissue engineering. ${ }^{54}$ Due to their inherent shape and distribution, they can effectively mimic the extracellular matrix (ECM). Thus, they are 
potential materials to achieve improved cellular growth and functional expression. ${ }^{55}$ As in the other types of scaffolds, natural or synthesized fibers are non-conductive. However, in this case, the electrical properties have only been integrated through $\mathrm{CP}$ coating, which can sometimes be a difficult task since the $\mathrm{CP}$ monomer prefers to polymerize in the liquid phase rather than on the fiber surface. ${ }^{56}$ In the few examples in the literature, the most common coating process used was chemical polymerization by immersing the $3 \mathrm{D}$ fibrous mesh in the corresponding monomer and oxidant solutions. ${ }^{56}$ We anticipate that any of the methods used to deposit CP onto 3D substrates described so far along this review, could also be applied in fibrous meshes. For example, Chen et al. reported an in situ interfacial polymerization of EDOT in ether on bacterial cellulose membranes previously immersed in aqueous PSS. ${ }^{57}$ Similarly, Yow et al. developed an interfacial polyelectrolyte complexation (IPC) technique, where the chemical polymerization occured at the interphase of aqueous solutions containing oppositely charged electrolytes. ${ }^{58}$

On the other hand, fibers are usually synthesized in films and the main goal is to achieve 3D fibrous meshes. Fibers are commonly synthesized via electrospinning and can be prepared as randomly dispersed or patterned meshes. Briefly, a high-voltage electrostatic field is applied to a polymer solution or melt until a charged liquid jet is formed and, as it flies towards a collector, the solvent is dried, resulting in homogeneous fibers with diameters of a few hundred nanometers. Although this methodology mainly produces membranes up to few micrometers in thickness, there exist scarce examples in the literature where 3D structures have been obtained using new and original strategies. Jin et al. manufactured a special collector to fabricate a 3D "fluffy" PPy-coated conductive fibrous PLLA scaffold, which consisted of a hemispheric plastic dish embedded with stainless-steel probes and covered with aluminum foil. ${ }^{59}$ In another study, Pelton and co-workers prepared a 3D fibrous scaffold by extrusion of a poly-96L/4D-lactide (PLA) filament and then 
manually carding the fibers obtained into sheets and punching them until a nonwoven scaffold of $2 \mathrm{~mm}$ thickness was obtained. ${ }^{60} \mathrm{In}$ a third example, Xu et al. used electrospinning to accumulate 3D polyacrylonitrile (PAN) nanofibers into a collector filled with ethanol, which was shaken every few minutes to ensure the homogeneity of the fibers along the 3D structure. ${ }^{61}$ In all these reports, PPy was in situ chemically polymerized by immersion of the 3D fibrous scaffold into the corresponding monomer and oxidant solution. ${ }^{14,59}$

Even though all the examples described first form the electrospun substrate before its $\mathrm{CP}$ coating, there exists one example in the literature where the electrospinning solution already contains a mixture of the biopolymer and the CP. Subramanian and co-workers were able to electrospin a solution of PLGA with PHT and collect 3D axially aligned nanofibers. The authors designed a collector as a grounded rotating mandrel, shown in Figure 5, with a small insulating gap dividing the rod into two independent-like electrodes, thus allowing the deposition of longitudinally oriented fibers. ${ }^{62}$

\section{Characterization of 3D materials}

\subsection{Common characterization of $3 D$ scaffolds}



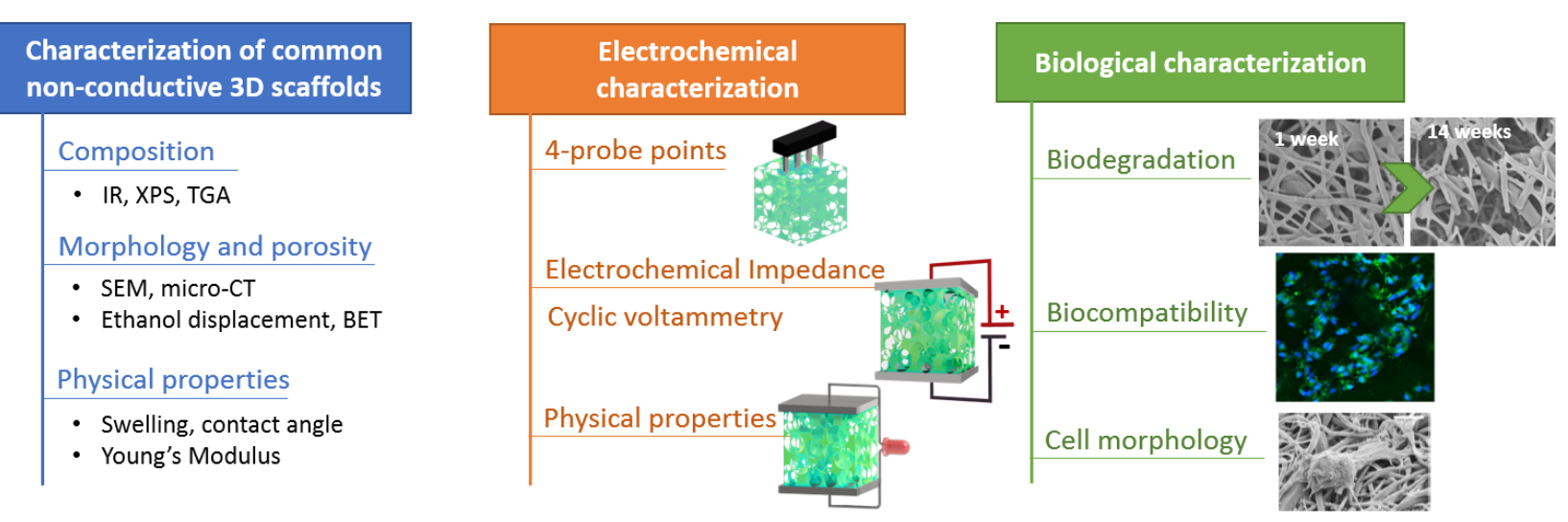

Figure 6. Characterization techniques employed for conductive 3D architectures. Adapted with permission from ref 63, copyright (2012) Springer; ref. 50, copyright (2016) American Chemical Society; ref 13, copyright (2009) Elsevier.

Scaffolds intended for biomedical purposes are designed for effective cell-scaffold contact and favor cellular attachment. Thus, analysis of the composition and the functional groups in the backbone of the polymeric matrix is essential to understand and guarantee a successful interaction. Fourier transform infrared spectroscopy (FTIR) is the most commonly used technique to determine the composition of the scaffolds, ${ }^{21-22}$ while XPS gives an accurate percentage of the chemical elements present in the materials. ${ }^{9}, 26$ Thermogravimetric analyses (TGA) can not only give information on the thermal stability of the entire scaffold, but also can quantify the percentage of each material within the matrix. CPs are highly thermally stable. ${ }^{64-66}$ Thus, the incorporation of $\mathrm{CP}$ into the polymeric structure can increase significantly the thermal decomposition temperature of the resulting substrates when compared to the bare natural polymer. ${ }^{21}$ Such an effect is observed when high amounts of $\mathrm{CP}$ are present and can be explained due to the high electrostatic interaction occurring between the two polymers. However, when the matrix is composed of less than $10 \%$ of $\mathrm{CP}$, it shows no effect on thermal stability. ${ }^{35,37,56}$

Scaffolds engineered for biological application, and in tissue engineering in particular, must have appropriate pore size to allow cellular infiltrations, and homogeneous interconnected porosity 
to enhance the medium and nutrient flow to the seeded cells. Macroporous substrates range from a few tens to several hundreds of micrometers, ${ }^{22,26,35}$ while fibers and hydrogels have a much smaller porosity, with sizes below a few tens of micrometers, what hinders the cellular penetration inside the tridimensional structure. ${ }^{7}{ }^{14}$ Moreover, the orientation of the pores plays a crucial role in determining the biological activity of the material. Patterned structures should be applied for tissues with a specific directionality, such as muscle cells, while randomly distributed porosity mimics tissues with undefined directionality, such as brain tissues. ${ }^{67-69}$ Scanning Electron Microscopy (SEM) is the most common method employed to analyze the internal and/or external morphology of the scaffolds. However, it can only examine 2D cross-sections of the entire assembly. ${ }^{21}$ To obtain the tridimensional visualization of the skeletal structure, micro-computed tomography $(\mu \mathrm{CT})$ can be a proper alternative. This non-destructive technique gives a detailed 3D model reconstruction of the entire scaffold. ${ }^{35}$ More detailed analyses on the surface area can be obtained from porosimetry techniques, such as ethanol displacement, ${ }^{21}$ Brunauer-Emmett-Teller (BET) ${ }^{37}$ or mercury porosimetry. ${ }^{25}$

Tridimensional porous scaffolds have porosities between $60 \%-90 \%, 26,35-36$ and the incorporation of the $\mathrm{CP}$ does not always have the same effect. Several studies have shown that the addition of $\mathrm{CP}$ decreases the overall porosity of the scaffolds. In other cases, the addition of the CP increases the porosity and the surface area. ${ }^{37}$ Yazdimamaghani et al. demonstrated a decrease in the pore size with the addition of PEDOT:PSS compared to a bare matrix of gelatin and bioactive glass, but a decrease in the size when the PEDOT:PSS concentration was raised. ${ }^{35-36}$

It is reasonable to expect thicker fibers after $\mathrm{CP}$ coating, resulting in smaller pore size. However, most reports did not observe a significant difference, likely since the coating layer is usually in the nanometric range. ${ }^{56}$ Furthermore, Chen et al. demonstrated that an excess of the PSS dopant within 
the conductive matrix may disturb the polymerization reaction and result in thinner fibers. ${ }^{57}$ In hydrogels materials, the pore size may be lower than in porous scaffolds, commonly below $20 \mu \mathrm{m}$. In this case, the pore size seems to be more dependent on the hydrogel polymer, addends and crosslinking level. ${ }^{70}$

In addition, $\mathrm{CP}$ coating formed characteristic nano- and microscale topography roughening the surface of the matrix (Figure 7). ${ }^{23}$ Less smooth surfaces can be directly related to the cell adhesion, thus improving cellular proliferation and, in some cases, differentiation. ${ }^{29}$ 
a)
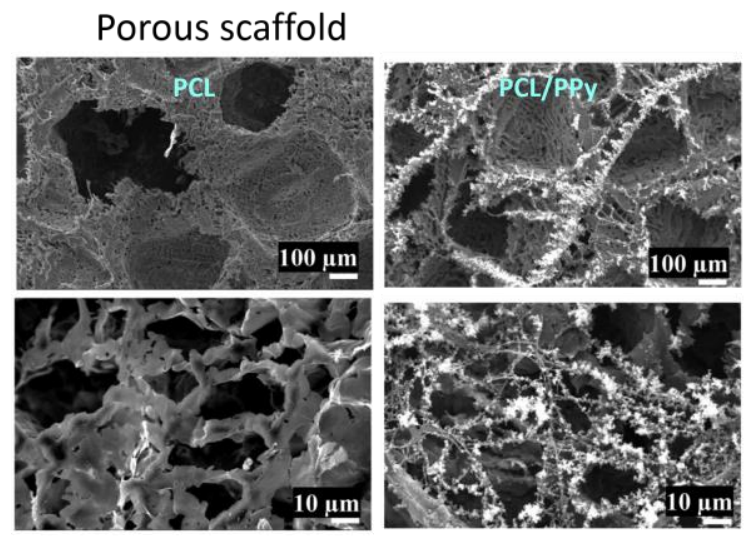

b) Hydrogel
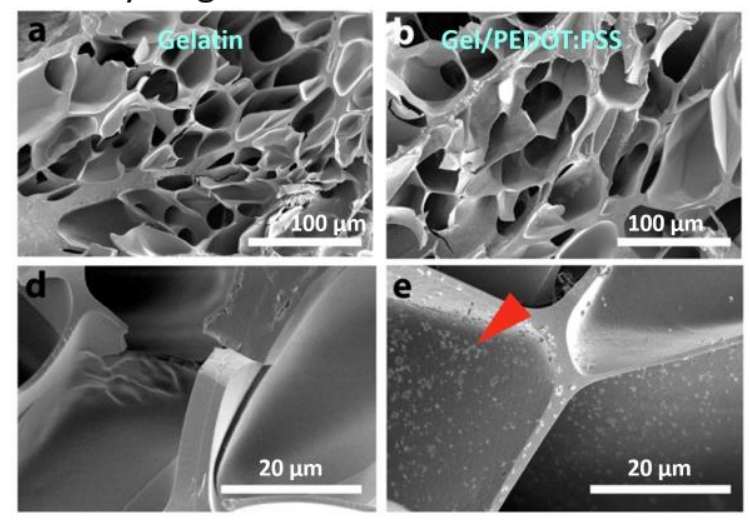

c) Fiber Meshes

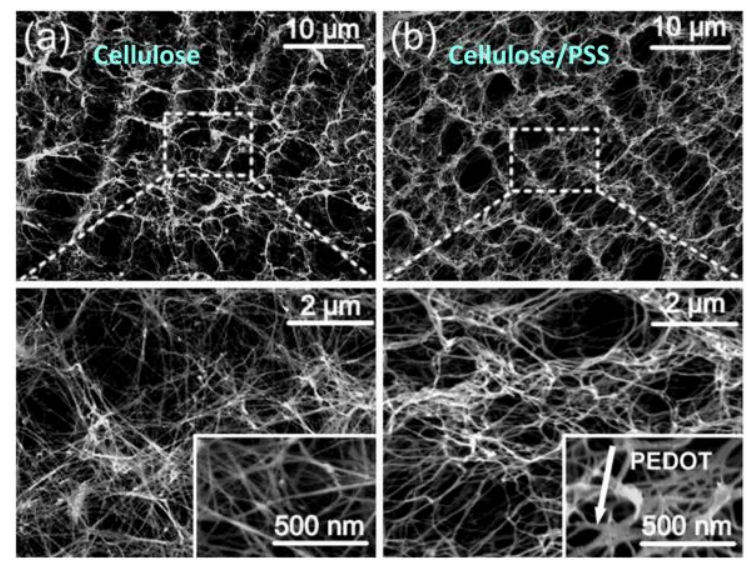

Figure 7. SEM images of a) PCL and PCL/PPy porous scaffolds, b) Gelatin and gelatin/PEDOT:PSS hydrogel and c) Bacterial nanocellulose (BC) and BC/PEDOT fibers. Adapted with permission from a) ref. 26, copyright (2016) Elsevier, b) ref. 71, copyright (2018) American Chemical Society, c) ref. 57, copyright (2015) Springer. 
Swelling analyses are extremely important for applications that include implant incorporation in in vivo experiments, such as tissue engineering or drug delivery. On the one hand, strong water uptake facilitates the migration and infiltration of the cells, the diffusion of the nutrients and waste removal. ${ }^{37}$ On the other hand, a huge and uncontrolled increase of the substrate can affect the surrounding living tissue and lead to severe inflammation. ${ }^{72-73}$ For porous or fibrous materials, the change in the volume is generally negligible and the swelling ability is directly correlated with the porosity of the system. ${ }^{35}$ It has been observed that a decrease in the porosity yields to a decrease in the water uptake. ${ }^{21}$ However, swelling behaviors of hydrogels are significantly affected by the composition and amount of hydrophilic groups present. ${ }^{70}$ Discrepancies between the different studies regarding the swelling effects when incorporating a CP are also described. Some reports observed a reduction in the swelling after incorporating CPs, concluding that the main factor governing this phenomenon was the hydrophobicity of the conjugated matrix. ${ }^{22,37,48,74}$ In order to reverse the hydrophobicity, some scientists have been altering the matrix composition to improve the swelling ability of conductive substrates. ${ }^{75}$ Mawad et al. obtained a higher ratio of swelling compared to previously reported conductive hydrogels by mixing functionalized PEDOT$\mathrm{COOH}$ with polyacrylic acid hydrogel. ${ }^{50}$ They determined that such an increase was due to the ionization at $\mathrm{pH}=7.4$ of the free carboxylic groups present in both polymers.

The Young Modulus can be determined using a compression mechanical testing apparatus or, in the case of hydrogels, rheology. The Young modulus is obtained by measurement of the elastic deformation response of the material against the applied force. The stiffer the material, the lower the elastic deformation. ${ }^{43,48-49}$ Although the Young Modulus can be altered by dopants, monomer concentrations, hydrophobic polymer content or crosslinking density, ${ }^{37,70,75}$ we will only focus on 
those alterations coming from the conjugated backbone. Usually, the compressive modulus in dry conditions is increased by the addition of CP into the matrix even at low concentrations of CP. ${ }^{24}$, 26, 32, 5835 This effect has been attributed to the highly conjugated, and thus rigid and stiff, nature of the CP. ${ }^{48,56,74}$ Furthermore, in the case of fibrous substrates, Niu and co-workers presumed that such an increase may also come from deposition within the interstices, which act as crosslinks and reduce the sliding of neighboring microfibers in contact with each other. ${ }^{56}$ Alternatively, Yang et al. hypothesized that clustered PPy within alginate hydrogels may be connected, thus strengthening the internal network with hydrogel portions. ${ }^{43}$

To determine the correct Young modulus, experiments must be performed in wet conditions, since the biological tissues are in aqueous media. Furthermore, the swelling can greatly affect the scaffold strength. Del Agua et al. observed a reduction of the half value after swelling compared to dry conditions. ${ }^{24}$ Wang et al. rationalized that this effect was due to the weakened matrix resistance to external compression caused by the presence of water molecules within the structure. ${ }^{74}$ The redox state of the conjugated polymer can also affect the mechanical strength of the material, which can be rationalized due to secondary bond formation within the structure, ${ }^{50}$ or even by electrostatic interaction between the $\mathrm{CP}$ and the non-conductive matrix. ${ }^{43}$

\subsection{Electrochemical characterization}

The most peculiar and specific characteristic of CPs is their ability to transport electrons through their conjugated bonds. This conductivity can be beneficial for electrophysiological-related bioapplications, such as neural or cardiac communication. It has been demonstrated that substrates with electrical properties have a positive effect on cellular differentiation. ${ }^{76-77}$ Moreover, conductivity is an indispensable property for a scaffold to be used as an electrode itself for both recording electrical activity in the biological material and electrically stimulating cells or tissue. 
The intensity current of biological processes in human body has been determined in the order of nanoamperes $;{ }^{78}$ thus, even semi-conductor materials are suitable for tissue regeneration applications. It is worth to noting that each study undertook experiments using different conditions, even for the same technique, which makes it challenging to compare the electrical conductivity values between them. Thus, we are reviewing studies under this qualified point of view.

The electrical properties are directly related to the amount of $\mathrm{CP}$ present within a substrate. Thus, a measure of the conductivity is a straightforward way to determine the composition of the 3D scaffold. There are several methods to measure the conductivity. (i) Direct measures that give an

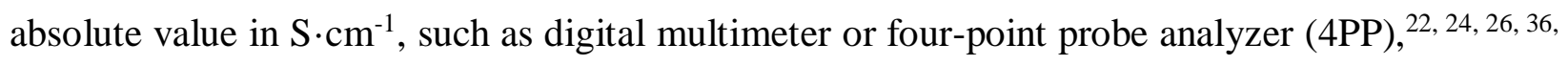
$45,56,74,79$ although these procedures are more common for 2D films, some scientists also use them for 3D substrates in direct contact with the scaffold or using two metal plate electrodes as support. ${ }^{18,43,63}$ (ii) Evaluation of the current passing through the scaffold while a controlled voltage range is applied, i.e., cyclic voltammetry, with an electrochemical station. ${ }^{28,57,70}$ (iii) Indirect measures through the analysis of the resistivity using electrochemical impedance spectroscopy (EIS), which results in the inverse of the conductivity. ${ }^{46,50}$ EIS presents the signal as a function of frequency at a constant potential and generates analyzable information at each frequency. This is a very powerful and sensitive tool to study the processes of sensing, cell attachment, and drug release. (iv) Some studies construct an electrical circuit that includes the scaffold, an LED bulb and a power supply connected in series to qualitatively confirm the pass of electricity through the scaffold. ${ }^{7,31,34}$ Positive results are obtained if the bulb lights up once the circuit is closed. (v) Finally, the distribution of surface current can be analyzed using a scanning electrochemical microscope (SECM). ${ }^{21}$ 
In all cases, whether in porous scaffolds, fibers or hydrogels, the addition of a small amount of CP increases the conductivity significantly. ${ }^{22,43,56}$ For example, Shahini et al. observed a $70 \%$ increase in conductivity in a scaffold containing only $0.3 \%$ of PEDOT. ${ }^{35-36}$ The method of fabrication is very important to the final properties. Some scientists find that the chemical polymerization method that results in the highest conductivity is the vapour phase polymerization, 7,29 although electropolymerization has been shown to produce higher conductivity. Severt and coworkers demonstrated that the electrical properties can be even more enhanced if, after chemical polymerization, a second coating layer is deposited via electrodeposition. ${ }^{18}$

Additionally, the type and amount of dopant or non-conjugated polymer present within the matrix can also have an impact on the electrical properties of the system. ${ }^{49,57,70}$ An excessive concentration of dopant can lead to 3D structural damage, as observed by Chen and co-workers, since it may disturb the polymerization deposition, thus leading to, for example, thinner coated layers. ${ }^{57}$ Moreover, Mawad et al. discovered that the CVs became more reversible after doping. ${ }^{50}$ As previously pointed with the evaluation of the mechanical properties, it is important to evaluate the conductivity in biological conditions, and, thus, samples should be hydrated when performing the measurements. It is important to evaluate the electrode-media interface, since it is the dominant factor in the electron transmission from the substrate to the media. ${ }^{75}$ In semiconductive matrices incorporating $\mathrm{CP}$, such as chitosan/gelatin hydrogels, it was observed that the conductive ability of the polymeric matrices is significantly enhanced in hydrated scaffolds, which can be associated with the electrolyte nature of the non-conjugated polymer. ${ }^{74}$ On the other hand, Niu and co-workers observed a decrease in the conductivity after several hours of cell culture. ${ }^{56}$ The authors detected that PEDOT/PLLA was associated with losses in the conductivity of $22 \%$ and $65 \%$ after $100 \mathrm{~h}$ and $240 \mathrm{~h}$ of incubation with cells, respectively, and this 
could be related to the biodegradability rate of the scaffold. Moreover, EIS can be also used for cell monitoring after seeding within the $3 \mathrm{D}$ scaffold to evaluate attachment and development. ${ }^{24,75}$

\subsection{Biological characterization: biocompatibility, biodegradability and cell imaging after culture}

Biocompatibility, i. e., a good cellular response to a material, is the first requirement a scaffold must comply with to be feasible for any kind of biological application. Even though the conjugated polymers have never shown any trace of cytotoxicity, each one of the studies discussed in these pages has been demonstrated to be biocompatible for a different kind of cell, including neurons, osteoblasts, cardiomyocytes, and mesenchymal stem cells, among others.

One of the questions that always arises when using synthetic materials in any biological application, particularly in long-term implants, is "how long will they last once are placed inside the body?". In tissue engineering, a suitable scaffold must be either reabsorbed by the biological system, becoming part of the tissue, or biodegrade, creating space for cellular growth and rearrangement of new tissue. ${ }^{74}$ The scaffold degradation rate should then match the new tissue formation rate for optimal regeneration. In summary, all implants must remain long enough to provide the desired support and/or connective effect, but no longer than necessary as they may become a hindrance to repair. ${ }^{80}$ Biodegradation refers to degradation occurring through disintegration, hydrolytic mechanisms or enzymatic activity. ${ }^{81}$ In practice, the biological degradability of 3D substrates is evaluated in vitro under physiological conditions. ${ }^{22,74}$ Usually, the presence of $\mathrm{CP}$ results in higher biodegradability resistance. ${ }^{48}$ For instance, the incorporation of $0.3 \%$ of PEDOT to gelatin-based porous substrates reduces the biodegradation from $70 \%$ after one day to less than $40 \%$ in 90 days. ${ }^{22,35}$ These observations have been be attributed to the presence of PEDOT nanoparticles on the scaffold surface and its increased hydrophobicity. The 
permeability and contact area between the water containing enzyme and the biopolymer is decreased, thus resulting in a significantly lower degradation rate. ${ }^{74}$ In functionalized monomers, such as PEDOT-COOH, the increase in stability can be rationalized with increased crosslinking coming from the formation of new covalent bonds between the carboxyl groups and the biopolymer. ${ }^{22}$

On the other hand, degradation of conductive substrates can occur due to delamination that typically occurs with CP coatings after time. To overcome this limitation, Severt and co-workers have developed a coating method where a second layer of CP is deposited electrochemically after a first chemical polymerization of the CP. ${ }^{18}$ In their work, they demonstrated that such a second layer enhanced the conductivity, stability and surface area of the CP coating on silk, thus avoiding delamination of the $\mathrm{CP}$.

Apart from the appropriate degradation rate, the degradation products of the scaffold should not be cytotoxic, so cell viability studies of the biodegradation solution is typically performed. ${ }^{74} \mathrm{In}$ addition, the scaffolds can be designed with side chains or dopants known to have no foreign body response, such as polyethylene glycol (PEG), poly (glycerol-sebacate) (PGS), and poly (tetrafluoroethylene) (PTFE) ${ }^{81}$

After in vitro experiments, the morphology of the cells as well as the degree of migration inside the substrate can be visualized using SEM in addition to the common staining analyses. ${ }^{61}$ However, after incubation, wet samples must be prepared for the SEM vacuum to avoid sudden modification of the cellular morphology. ${ }^{14}$ The resulting images may show cells covering the surface of the substrate, which is an indicator of strong adhesion and biocompatibility. And, usually, such coverage is larger with CP-coated substrates, which can be attributed to an increase of the conductivity of the scaffold, which is beneficial for those cells with electrical connections, such as 
neurons and cardiomyocytes, or due to an increase in the roughness of the surface. ${ }^{74}$ It is worth noting that the morphology of cells grown in 3D incubation can differ from those grown on flat surfaces, i. e., cell culture dishes, and can also be dependent on the kind of substrate, composition or rugosity. 7,36

\section{Biological applications of 3D scaffolds based on conductive polymers}

Conductive 3D scaffolds have demonstrated great potential for a wide range of biomedical applications, such as tissue engineering, drug delivery and biosensing, due to their excellent morphological, physical, electrical and biological properties (Table 1). This section focuses on specific examples that employ 3D scaffolds in biological applications, summarized in Figure 8.

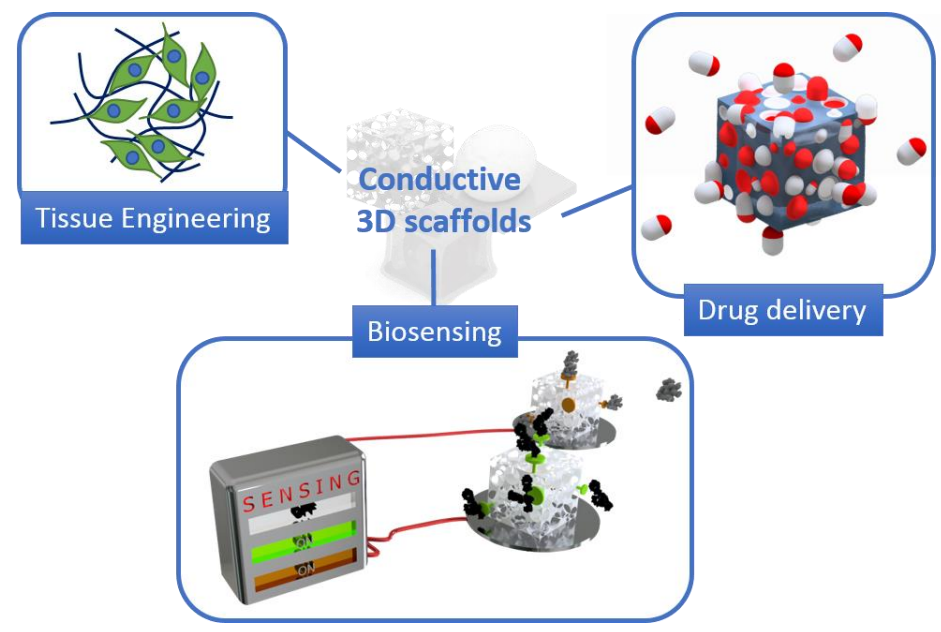

Figure 8. Biological applications where 3D scaffolds have been applied. 
Table 1. Summary of the of the CP-based structures, properties and biological application highlighted in this article.

\begin{tabular}{|c|c|c|c|c|c|c|c|c|c|}
\hline Structure & Composition & Fabrication method & $\begin{array}{l}\text { Max. pore } \\
\text { size }\end{array}$ & $\begin{array}{l}\text { Conductivity / } \\
\text { resistance }\end{array}$ & $\begin{array}{l}\text { Swelling } \\
\text { ratio }\end{array}$ & $\begin{array}{l}\mathbf{Y M} \\
(\mathbf{k P a})\end{array}$ & Biodegradability & Biomedical Application & Ref. \\
\hline \multirow[t]{18}{*}{ Porous } & Melamine/PEDOT/PSS & Immersion & $200 \mu \mathrm{m}$ & Stress dependent & - & - & - & Pressure sensor & 34 \\
\hline & PPy-Alg/chitosan & Immersion & $100 \mu \mathrm{m}$ & $1000 \mathrm{nA}$ & $9 \%$ & - & $30 \%$ after 4 weeks & Tissue engineering & 21 \\
\hline & $\mathrm{Cs} / \mathrm{Gel} / \mathrm{PEDOT}$ & Immersion & $250 \mu \mathrm{m}$ & $10^{-1} \mathrm{~S} / \mathrm{cm}$ & $400 \%$ & 30 & $37 \%$ after 8 weeks & Tissue engineering & 74 \\
\hline & PPy/Silk and PEDOT/Silk & Immersion $+\mathrm{EP}$ & $5 \mu \mathrm{m}$ & $10^{2}-10^{3} \Omega / \mathrm{sq}$ & - & - & - & - & 18 \\
\hline & $\mathrm{rGO} / \mathrm{PPy} / \mathrm{CPP}$ & Immersion & $200 \mu \mathrm{m}$ & - & - & $186 \mathrm{MPa}$ & - & Stem cells differentiation & 9 \\
\hline & PTMC/PPy & Immersion & $250 \mu \mathrm{m}$ & $1 \mathrm{k} \Omega / \mathrm{sq}$ & - & - & - & Stem cells differentiation & 27 \\
\hline & $\mathrm{PPy} / \mathrm{PCL}$ & Immersion & $328 \mu \mathrm{m}$ & $10^{-1} \mathrm{~S} / \mathrm{cm}$ & - & $12 \mathrm{MPa}$ & $2 \%$ after 10 weeks & Tissue engineering & 26 \\
\hline & PEDOT/PS and PPy/PS & VPP & $150 \mu \mathrm{m}$ & - & - & - & - & Tissue engineering & 7 \\
\hline & $\mathrm{PEDOT} / \mathrm{SiO}_{2}$ & VPP & $>100 \mu \mathrm{m}$ & $0.5 \mathrm{k} \Omega$ & - & - & - & - & 31 \\
\hline & PEDOT/PCL & VPP & $600 \mu \mathrm{m}$ & - & - & $10^{3}$ & - & Stem cells differentiation & 29 \\
\hline & PEDOT-HA/Cs/Gel & Freeze-drying & $300 \mu \mathrm{m}$ & $10^{-3} \mathrm{~S} / \mathrm{cm}$ & $700 \%$ & 55 & $43 \%$ after 8 weeks & Tissue engineering & 22,23 \\
\hline & PEDOT/XantamGum & Freeze-drying & $150 \mu \mathrm{m}$ & $10^{-2} \mathrm{~S} / \mathrm{cm}$ & $400 \%$ & $<40$ & - & Live-cell monitoring & 24 \\
\hline & Gel/PEDOT/PSS & Freeze-drying & $300 \mu \mathrm{m}$ & $210 \mu \mathrm{S} / \mathrm{m}$ & - & - & - & Tissue engineering & 36 \\
\hline & PEDOT/PSS/GOPS & Freeze-drying & $40 \mu \mathrm{m}$ & $10^{1} \mathrm{k} \Omega$ & - & 4.5 & - & Tissue engineering & 25 \\
\hline & Gel/BaG/PEDOT/PSS & Freeze-drying & $250 \mu \mathrm{m}$ & $170 \mu \mathrm{S} / \mathrm{m}$ & $240 \%$ & $35 \mathrm{MPa}$ & $35 \%$ after 90 days & Stem cells differentiation & 35 \\
\hline & HAp/Gel/Si/PPy & Freeze-drying & $350 \mu \mathrm{m}$ & - & $250 \%$ & $110 \mathrm{MPa}$ & - & $\begin{array}{l}\text { Tissue engineering, drug } \\
\text { delivery }\end{array}$ & 37 \\
\hline & $\mathrm{PANi} / \mathrm{CNT}$ & $\mathrm{EP}$ & $1 \mu \mathrm{m}$ & $186 \mathrm{~S} / \mathrm{m}$ & - & - & - & - & 33 \\
\hline & rGO/PPy/HAp & $\mathrm{EP}$ & $400 \mu \mathrm{m}$ & - & - & $186 \mathrm{MPa}$ & - & Tissue engineering & 32 \\
\hline \multirow[t]{11}{*}{ Hydrogel } & Collagen/PPy & Ox. crosslinking & - & $3.4 \times 10^{-3} \mathrm{~S} / \mathrm{cm}$ & & $1-3$ & - & Glucose sensing & 47 \\
\hline & HA/CNT/PPy & Ox. crosslinking & - & $12.6 \mathrm{k} \Omega / \mathrm{sq}$ & $-30 \%$ & 3.2 & $100 \%$ after 2 days & Stem cells differentiation & 48 \\
\hline & $\mathrm{Alg} / \mathrm{PPy}$ & Filling & $1 \mu \mathrm{m}$ & $31.1 \times 10^{-4} \mathrm{~S} / \mathrm{cm}$ & & $\sim 2000$ & - & Stem cells differentiation & 43 \\
\hline & CMCS/PEDOT & Filling & $100 \mu \mathrm{m}$ & $4.7 \times 10^{-3} \mathrm{~S} / \mathrm{cm}$ & $1700 \%$ & 12.5 & $35 \%$ after 10 weeks & Tissue engineering & 45 \\
\hline & GelMa/PEDOT/PSS & Filling & $50 \mu \mathrm{m}$ & $261 \mathrm{k} \Omega / \mathrm{sq}$ & $600 \%$ & 7.6 & $25 \%$ after 2 weeks & Tissue engineering & 71 \\
\hline & $\begin{array}{l}\text { PNAGA-PAMPS/ } \\
\text { PEDOT/PSS }\end{array}$ & Polym. before gelation & - & $0.2-2.2 \mathrm{~S} / \mathrm{cm}$ & $65-88 \%$ & $30-110$ & - & Inks for $3 \mathrm{D}$ printing & 49 \\
\hline & HEMA/PAA/PEDOT & Polym. before gelation & $30 \mu \mathrm{m}$ & - & $\sim 2500 \%$ & $\sim 100$ & - & Tissue engineering & 70 \\
\hline & Collagen/PEDOT/PSS & Polym. before gelation & $50 \mu \mathrm{m}$ & $28 \mathrm{k} \Omega / \mathrm{sq}$ & - & 45 & - & Live-cell monitoring & 75 \\
\hline & $\begin{array}{l}\text { PAA/PEG-DA/ } \\
\text { APMA-PEDOT }\end{array}$ & Polym. before gelation & - & - & $\sim 5000 \%$ & $\sim 45$ & - & Tissue engineering & 50 \\
\hline & PVA-hep/PEDOT & Filling & - & $\sim 50 \Omega / \mathrm{sq}$ & - & - & - & Drug delivery & 46 \\
\hline & PTAA & Side-chain crosslinking & $50 \mu \mathrm{m}$ & $\sim 10^{-5} \mathrm{~S} / \mathrm{cm}$ & $300-800 \%$ & - & - & Tissue engineering & 52 \\
\hline \multirow[t]{5}{*}{ Fibers } & PLA/PEDOT & Immersion & - & $0.4 \mathrm{k} \Omega / \mathrm{sq}$ & - & 15.7 & - & Tissue engineering & 56 \\
\hline & PAN/PPy & Immersion & $30 \mu \mathrm{m}$ & - & - & - & - & Tissue engineering & 61 \\
\hline & PLA/PPy & Immersion & $200 \mu \mathrm{m}$ & $1700 \Omega / \mathrm{sq}$ & - & - & - & Stem cells differentiation & 60 \\
\hline & PLLA/PPy & Immersion & $100 \mu \mathrm{m}$ & - & - & - & - & Tissue engineering & $14,{ }^{59}$ \\
\hline & Cellulose/PEDOT/PSS & Interfacial polym. & $5 \mu \mathrm{m}$ & $0.1 \mathrm{~S} / \mathrm{m}$ & - & 990 & - & - & \\
\hline
\end{tabular}




\subsection{Tissue engineering}

Most of the published works incorporating CPs in porous materials have been designed for Tissue Engineering applications. ${ }^{82}$ However, the majority are not tridimensional materials and have uneven nanoscale porosity, thus inducing the non-uniform deposition of the biological cells or tissue. ${ }^{32}$ Tissue regeneration aims to repair or replace damaged or diseased tissues with synthetic and/or natural implants. Those implants should mimic the environment of the host tissue to avoid possible rejection, and 3D scaffolds are the best choice for that purpose due to their lowcost, high reproducibility and controllable processing morphology and composition that can affect cellular growth and morphology. ${ }^{70}$ All these features have been shown to affect the cellular growth and morphology, as shown in Figure $9 .{ }^{61}$ Furthermore, the possibility of incorporating chemical groups, bioactive agents such as drugs, growth factors and dopants make them tunable to enhance the cellular adhesion and proliferation. ${ }^{75,83}$ For instance, the incorporation of hydroxyapatite (HAp), known as a bone mineral, shows strong adhesion and excellent proliferation with mouse MC3T3-E1 pre-osteoblasts. ${ }^{32}$ 


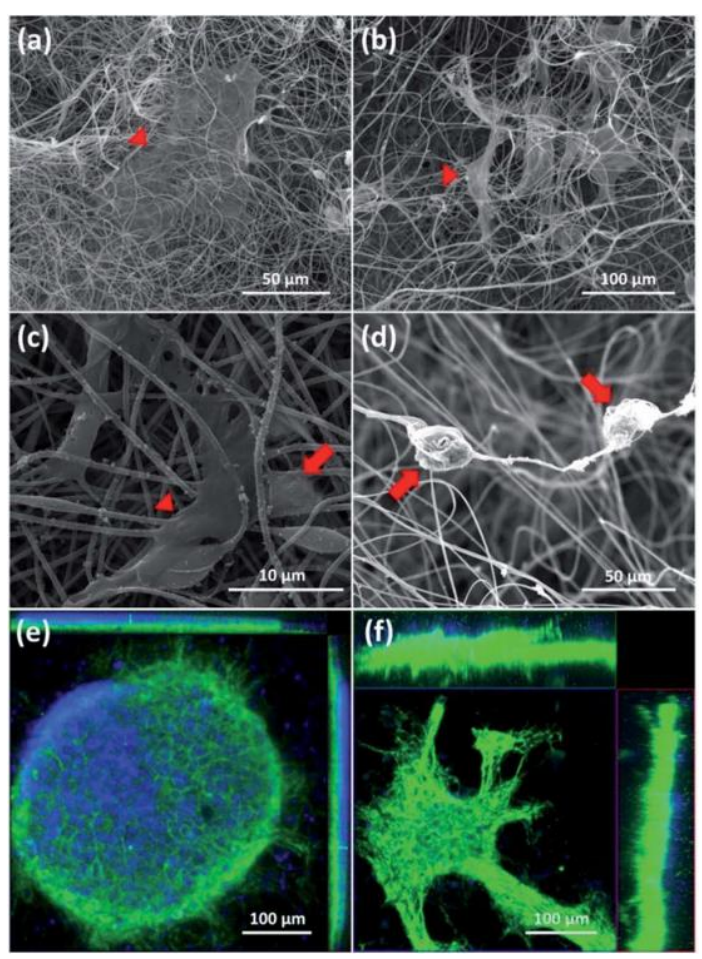

Figure 9. SEM of cortical cells cultured in 2D-CNFs (a and c), 3D-CNFs (band d) and confocal microscopic ortho-images of cell culture in 2DCNFs (e) and 3D-CNFs (f). The red arrows and triangles indicated two different cell morphologies respectively. Green: MAP2; blue: DAPI. Reprinted with permission from ref. 61, Copyright (2018) The Royal Society of Chemistry.

Tissue engineering can be understood as an implantation of a bare scaffold, with all the components to ensure its functionality, or an artificial tissue developed in vitro from the growth and invasion of cells inside a scaffold. Currently, regeneration employing 3D CP-incorporating scaffolds is in its early stages of investigation and there have not yet been published studies in which real in vivo implantation or full artificial tissue was developed. However, in vivo subcutaneous implantation of PPy/Alg hydrogels for eight weeks has been undertaken, and moderate immune reactions were induced. ${ }^{43}$ 
In practice, the studies are based on the 'healthy' growth of the cells of interest within the 3D structure, imaging their morphology and dispersion along the surface with SEM and fluorescent microscopy ${ }^{61}$ In few cases, the evaluation of characteristic cell functions have also been used to analyze cell proliferation. For instance, the concentration of vascular endothelial growth factor (VEGF) in 3T3-L1s fibroblasts, a stromal model, was characterized to quantify its pro-angiogenic potential. ${ }^{25}$ In addition, neurotransmitters and other specific protein markers for neurons and heart cells and have been utilized to determine cell proliferation. ${ }^{19,23,50}$ Inal and co-workers, have taken advantage of the effect of cells on the electrical conductivity of porous structures and used in situ impedance measurements as a tool to monitor cell growth. ${ }^{75}$ Moreover, phenotyping and gene expression characterization have become useful tools to follow up cellular behavior studies. ${ }^{22,74}$

In general, the results obtained predict the suitability of the scaffold employed for future applied tissue regeneration. One of the main requirements of a scaffold employed for regeneration purposes is its ability to maintain healthy cells inside its structure for long periods of time. All the works summarized in this section performed cellular cultures of at least 5 days and up to 21 days. The longer the study duration, the more unquestionable is the suitability of the scaffold for tissue engineering. In the literature, three main types of cells have been tested, all of which have electrically active properties: bone, neuronal and heart cells. The articles related to bone tissue engineering used pre-osteoblasts, i. e., mesenchymal stem cells that differentiate into bone cells. These will be further discussed in the 'Stem cell differentiation' section. The most employed neural model is the rat phaeochromocytoma-derived (PC12) cells. ${ }^{22,40,45,74}$ Neonatal rat ventricular cardiomyocites and mouse myoblasts $\mathrm{C} 2 \mathrm{C} 12$ cells have also been analyzed as models for heart regeneration. ${ }^{14,50,70}$ Other kinds of non-conductive cells that have been employed as models for 
cell viability and proliferative ability of 3D architectures include epithelial cells ${ }^{24}$ and fibroblasts. ${ }^{75}$

\subsubsection{Biomineralization for bone tissue engineering}

The biological integration with the surrounding tissue is what determines the potential of a scaffold in tissue regeneration. In bone engineering, a reliable way to the mimic the bone regeneration and evaluate the osteointegration is through in vitro mineralization of the substrate. In this process, the material is immersed in saturated simulated body fluid (SBF), a supersaturated calcium phosphate solution with composition similar to human plasma, for several periods at $37^{\circ} \mathrm{C}$ and the formation and rate of the deposition of bioactive minerals, such as hydroxyapatite (HA), on the surface is evaluated with SEM, EDX and Alizarin Red staining, a specific staining for HA (see Figure 10). ${ }^{20}$ Those minerals are similar to the ones present in the bone; thus, the faster the HA formation rate, the more feasible and stronger the osteointegration of the scaffold with the host tissue. Although the mineral covers the entire surface, it is essential to determine the existence of pores afterwards to ensure proper cellular penetration and nutrient and media flow. ${ }^{9,36-37}$
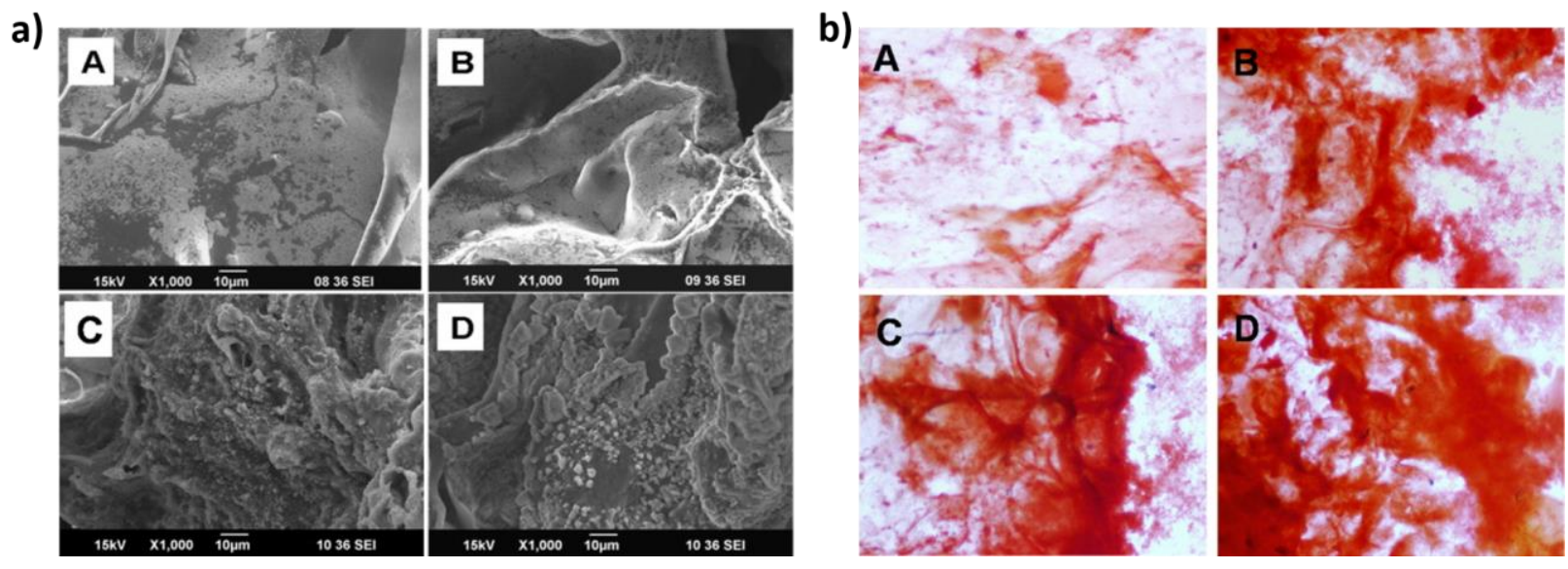

Figure 10. a) SEM images and b) Alizarin Red staining showing mineralization of chitosan on (A) $7^{\text {th }}$ and (C) 14th day, and chitosan/PPy-Alg on (B) 7th and (D) 14th day. Adapted with permission from ref. 21. Copyright (2013) Elsevier. 


\subsubsection{Stem cells differentiation}

In tissue engineering, one of the mechanisms used to develop artificial tissues in the laboratory is direct differentiation of pluripotent or mesenchymal stem cells inside a 3D structure. Growth factors, specific proteins and culture media are the features used to guide the differentiation into the desired cell type. However, the mechanical properties of the materials also play an important role in such differentiation. ${ }^{75}$

Human mesenchymal stem cells (hMSC) are the most frequently used cells for differentiation purposes. They are mainly extracted from the bone marrow, and can be guided to produce bone, cartilage and fat, as well as neuronal and endodermal lineages. ${ }^{29,} 35-36,43,60,84$ MC3T3-E1 mesenchymal cells derived from mouse skull have been used for osteoblast differentiation, and shown to form calcified bone tissue in vitro. ${ }^{7,9}$ Neural stem cells (NSCs) are self-renewing, multipotent cells used to generate neurons, astrocytes, and oligodendrocytes. ${ }^{23,}{ }^{48}$ Heart tissue differentiation has been achieved through $\mathrm{C} 2 \mathrm{C} 12$ cells, a mouse myoblast cell line commonly used as an in vitro model to study muscle differentiation..$^{50}$

\subsubsection{Electric stimulation}

One of the main advantages in the use of conductive scaffolds is that they can directly stimulate electrically conductive cells and tissues, providing a new tool that can interact electrically with biological systems. ${ }^{56}$ Several studies have observed that cellular regeneration of electroactive tissues is improved with local electrical stimulation (ES) of cells in scaffolds, which enhances the cell-to-cell and cell-to substrate interaction. ${ }^{85}$ ES has become a cheaper and better controlled alternative to the use of the bioactive molecules to modulate cell adhesion, migration, protein secretion and DNA synthesis, thus favoring and accelerating the proliferation and healing

process ${ }^{84}$ Clinically, application of pulsed electromagnetic fields has been employed to treat bone 
fractures and enhance spinal fusions. ${ }^{86}$ Now, the combination of CP scaffolds with ES opens a safer window for the application of localized and guided regeneration: the applied current passes through the scaffold structure, thus minimizing the electrolysis of the biological medium and reducing the production of cytotoxic agents. ${ }^{26}$

Zhang and co-workers demonstrated that the application of $200 \mu \mathrm{A}$ (4h per day, 21 days) on human adipose-derived mesenchymal stem cells (ADM-SCs) cultured in PPY/polycaprolactone (PCL) porous scaffolds promoted cell migration into deep regions of the scaffold and enhanced their osteogenic differentiation. ${ }^{26}$ Furthermore, ES on neurons and glial cell cultures has been demonstrated to reduce the formation of clusters in the PPy-coated structure only when applied at the beginning of culture, while improving the proliferation and acceleration of neuron maturation. ${ }^{61}$ Björninen et al. used an ES strategy to differentiate adipose stem cells (ASCs) into smooth muscle cells (SMCs). They manufactured a novel device to stimulate $243 \mathrm{D}$ scaffolds simultaneously over long periods. The PPy coating was shown to improve the SMCs expression and proliferation (see Figure 11). ${ }^{27}$ 


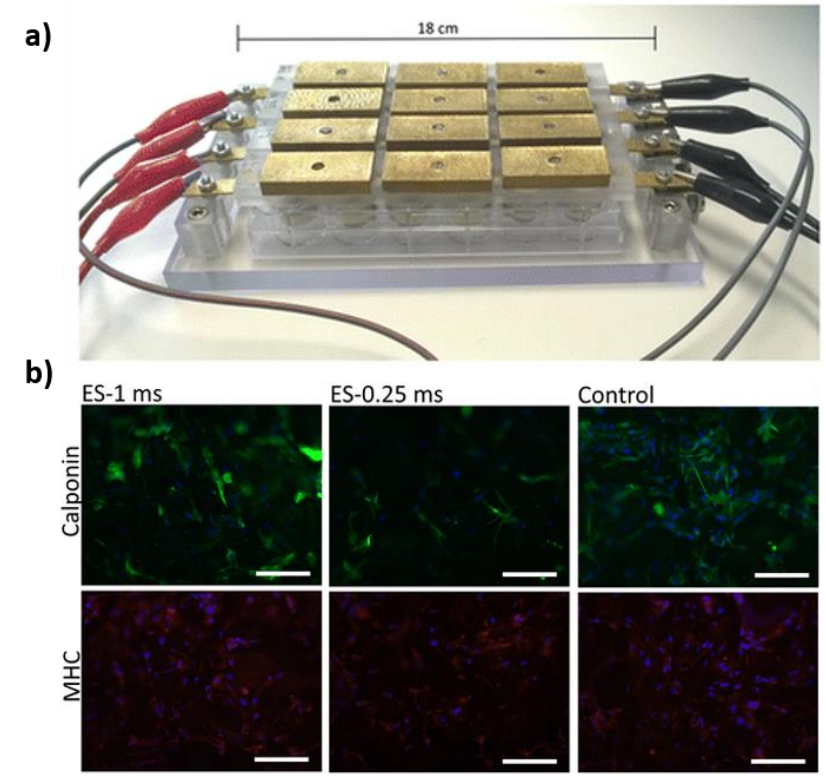

Figure 11. a) Assembly for $3 \mathrm{D}$ electrical stimulation device on $24-w e l l$ plate. b) Comparison of smooth muscle cells immunomarker expression in unstimulated controls and two ES paradigms at day 14. Scale bars represent $200 \mu \mathrm{m}$. Adapted with permission from ref. 27. Copyright (2016) Springer.

Although electrical stimulation not always has a significant effect in cell cultures, there are no reports of possible damage in the cellular culture. ${ }^{7}$ Furthermore, CPs-based devices loaded with drugs can be electrically stimulated for a controlled released.

\subsection{Drug delivery}

The combination of TE with drug delivery might be, in our minds, the ultimate treatment for several diseases. However, the combination of conductive polymers with drug delivery is in its early stage, and very few studies have been published in the past years. The mechanical properties play an important role in the drug release. The scaffolds should have a similar young modulus as the host tissue to ensure a prolonged release; otherwise, only short-term drug liberation can happen. Zanjanizadeh Ezazi et al. developed PPy/gel 3D scaffolds and loaded a model antibiotic, 
vancomycin (VCM), for anti-infection effect in bone regeneration purposes. The resulting scaffolds, with elasticity in the range of the cancellous bone, showed great potential to release drugs for 4 months, as anticipated by the authors. ${ }^{37}$ Vishnoi and co-workers developed a chitosan/gelatin/PPy scaffold and loaded alpha-ketoglutarate $(\alpha-\mathrm{KG})$, an intermediate in the formation of ATP in the citric acid cycle, to improve the neuro-2a cell proliferation. The aim of their work was to explore the exposure of neuronal cells to different amounts of drug in the same period through different delivery methods: exogeneous, 2D and 3D systems with and without PPy, concluding that the $3 \mathrm{D}$ system provides longer duration release of the drug. ${ }^{19}$ In addition, the liberation of a nerve growth factor (NGF) loaded in a conductive hydrogel scaffold was tested for PC12 cells, which are known to differentiate into neurons in response to NFG uptake. The results collected in Figure 12 showed a larger neurite outgrowth for the scaffolds loaded with NGF than for those unloaded, demonstrating the drug delivery ability of the material. ${ }^{46}$

a)

b)
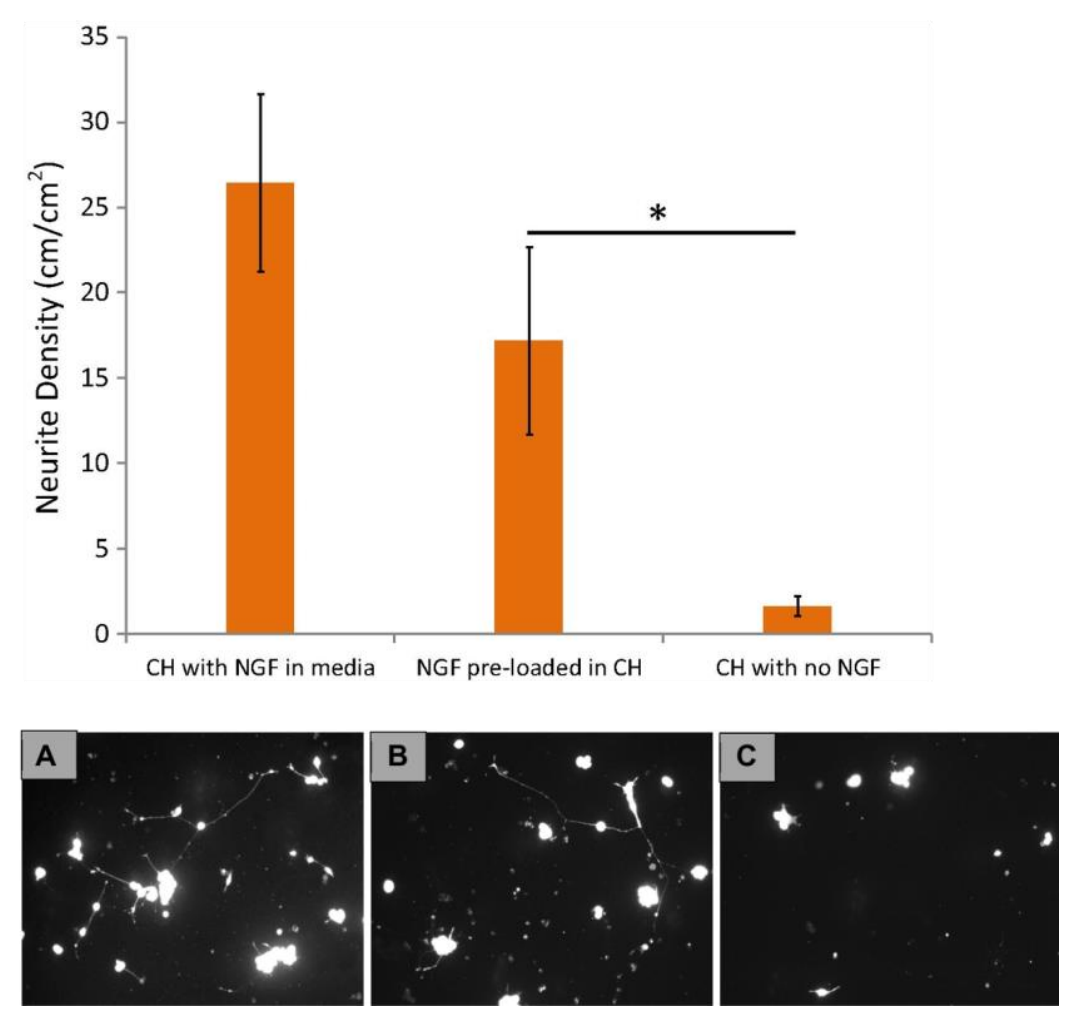
Figure 12. a) Neurite outgrowth response in PEDOT/PVA-Hep hybrids. b) Neurite outgrowth observed on samples after $96 \mathrm{~h}$ of incubation on (A) $\mathrm{CH}$ with NGF in media, (B) $\mathrm{CH}$ with NGF preloaded in the polymer and (C) no NGF in either media or polymer. Reprinted with permission from ref. 46. Copyright (2014) Elsevier.

There is clear evidence that the incorporation of CP into a non-conjugated matrix has a positive effect in regulating drug release: rougher surfaces generated by the $\mathrm{CP}$ coating increases the surface and thus the drug-polymer interaction. Moreover, the entrapment within the matrix and porosity of the substrate also have significant effects on the rate of release, being faster with larger pore sizes. ${ }^{37}$

\subsubsection{Drug loading and release characterization}

Drugs are usually incorporated at the initial steps of the processing by dispersion with the nonconjugated polymer, before the addition of the $\mathrm{CP}$ and any crosslinking. An initial loading is then calculated from the UV spectrometry of the supernatant of the centrifuged suspension. Since the drug is not usually covalently attached to the polymer backbone, subsequent processing steps and washing results in drug loss and reduction of the final loading. The release was tested similarly with UV spectrometry after incubation of the scaffolds in $\mathrm{PBS}$ at $37^{\circ} \mathrm{C}$ for long periods by measuring the absorbance of the supernatant at different time points. ${ }^{19,37}$ The same volume of PBS was added in the incubation tubes to maintain a constant final release volume.

\subsection{Biosensing}

Electrophysiology involves recordings from single ion channel protein, such as patch clamp or fluorescent techniques, to whole organ monitoring, as electroencephalogram or electrocardiography. For this reason, it is mandatory to used conductive materials to transfer the electric signals from the biological material to the monitoring system. The most common 
electrodes used in clinics nowadays are composed of platinum, silver, tungsten and iridium metals ${ }^{87}$ which can lead to biocompatibility issues due to the formation of insulating scars after short periods of post-implantation. Conjugated polymers have been largely used as coatings for implantable sensing devices to provide electrical conductivity, but also to improve the biocompatibility and avoid the scar formation. ${ }^{88}$ The inherent characteristic of conductive 3D architectures to be used as electrodes makes interesting materials for biosensing devices. ${ }^{89}$ In addition, due to their porous structure, 3D substrates have a much larger surface area for cell and biomolecule attachment, leading to higher biosensing sensitivity and concentration range than the bidimensional architectures. Ravichandran developed the first injectable electrochemical sensor for in situ monitoring glucose levels in tissue. ${ }^{47}$ The authors combined the hydrogel properties of collagen with the electronic functionality of PPy to manufacture the soft device. Glucose oxidase was then encapsulated within the gel matrix as the bioactive molecule of the sensor, allowing measurement of chronoamperometrically levels of glucose up to $10 \mathrm{mM}$. They were able to demonstrate its potential in vitro when administered in porcine meat tissue over 5 days. According to the authors, the main advantages of the developed sensor is that the hydrogel can adapt its conformation to the host tissue and provide a well-attached interface in dimensions as small as the puncture wound, thus reducing damaging invasiveness. ${ }^{47}$ Cysewska manufactured also a glucose sensor by electrodeposition of PPy in a Pt surface. The authors obtained non-porous structures with PPy particles with sizes up to the milimiter scale by controlling the deposition charge of the method. ${ }^{89}$

Another example is the piezoresistant pressure sensor developed by Ding et al. to monitor human motions for ultimate application in robotics, artificial intelligence and personal healthcare monitoring. ${ }^{34}$ The device was composed of a melamine sponge coated with PEDOT/PSS solution. 
It demonstrated high compressibility, sensitivity and stability when tested with a volunteer's throat, index finger, elbow, and knee, as shown in Figure 13.
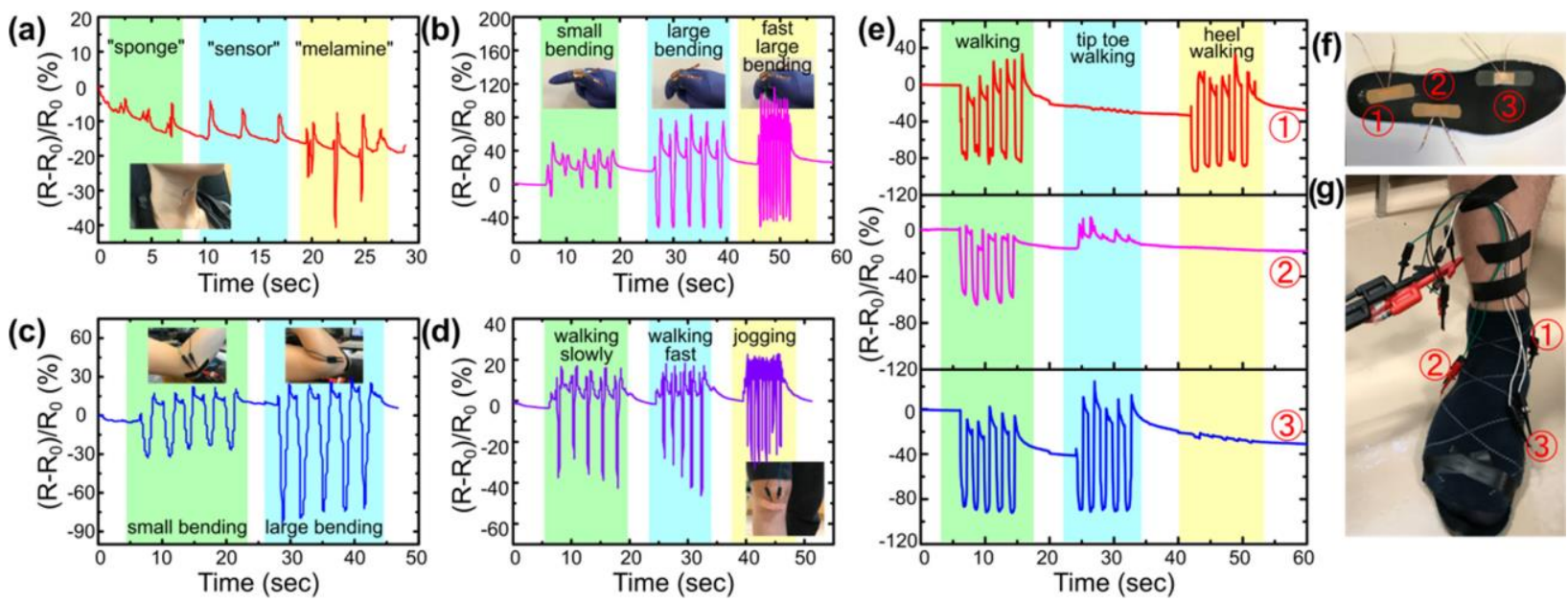

Figure 13. Applications of the PEDOT:PSS@MS pressure sensor for human motion detection: relative resistance variations of the sensor attached on a) the throat, b) the index finger, c) the elbow, d) the knee joint, (e-g) to an insole. Reprinted with permission from ref. 34. Copyright (2018) American Chemical society.

However, biosensors can be used in in vitro monitoring as well. Inal et al. manufactured a livecell monitoring platform based on microporous scaffolds composed of crosslinked PEDOT/PSS porous subtrates with freeze-drying. The scaffold was housed inside disposable cuvettes with a plastic tube incorporated and its potential for cellullar hosting and 3D culture monitoring was demonstrated. ${ }^{75}$

\section{Conclusions and future perspectives}

Conductive 3D substrates have emerged as the next generation platforms to translate the inlaboratory experiments to the real in vivo, thus providing a cost-effective reliable tool to predict cellular behaviors and responses before subsequent clinical trials. However, manufacturing the 
third dimension is not always an easy task, since CPs are insoluble, and their layer-by-layer stacking made them difficult to manipulate, which reduces the volume of publications even though new fabrication methodologies and techniques to achieve the 3D architecture have recently been developed. Here, we present a comprehensive overview of the state-of-the-art processing methods used, the characterization techniques necessary to analyze correctly the tridimensional properties and further biomedical applications tested for the conductive 3D substrates so far. Nevertheless, this field is in its early stages and we anticipate that it will be exponentially expanded in the coming years, and new methodologies to achieve the $3 \mathrm{D}$ architecture will be developed to meet the demands of clinical trials.

In that line, $3 \mathrm{D}$ printing is arising as a promising technique to automate and increase the bulk production of 3D scaffold fabrication. ${ }^{90}$ In contrast to conventional techniques, 3D printing allows the fabrication of customized scaffolds with controlled shape, pore size and pore structure through a precise layer-by-layer deposition. Some studies have used the 3D printer to generate a template that was then coated with the CP. ${ }^{29,91}$ Thus, we anticipate that the future lies with the preparation of conductive printable inks that can be used to construct the $3 \mathrm{D}$ scaffold in situ. ${ }^{49,92}$ Although not reviewed here, other studies have succeeded in the construction of a 3D scaffold by using carbon nanotubes or graphene instead of CPs to provide conductivitiy. ${ }^{79}$

All the biomedical areas of application require 3D scaffolds that mimic as close as possible the in vivo tissue. To ensure their adaptability, the substrates should obey the following criteria: contain homogeneous porosity with pore sizes large enough to host the cells, and to provide large surface area for cell attachment and proliferation; have similar mechanical properties to the tissue, including elastic modulus and wettability; and have a suitable surface composition to enhance the cell-substrate interaction. Thus, the design of the scaffold and all the steps during its manufacture, 
including its shape, composition and doping, must be developed with the final desired application in mind.

The main areas were conductive 3D scaffolds have the most potential are in tissue engineering of electroactive tissue, such as neurons, bones and heart cells, drug delivery and biosensing. In any of these applications, localized electric stimulation can play an important role in any of the cellular or tissue incubation steps. Initial studies demonstrated its potential, but further and extended investigations are in demand. Furthermore, the same implanted scaffold can be imagined as a single platform that can complete the three purposes at the same time. For instance, loaded substrates with drugs or growth factors and receptors could be used as monitoring devices for the regeneration of cells during their growth inside the scaffold, which could be enhanced with the loaded biomaterial. Nevertheless, the current studies are focused on in vitro assays that, although they are suitable for an initial prediction, await the translation to real cases.

\section{AUTHOR INFORMATION}

\section{Corresponding Author}

- NA. E-mail: nuria.alegret@ehu.es

- DM. E-mail: david.mecerreyes@ehu.es

\section{Author Contributions}

The manuscript was written through contributions of all authors. All authors have given approval to the final version of the manuscript.

\section{Funding Sources}

This project has received funding from the European Union's Horizon 2020 research and innovation programme under the Marie Sklodowska-Curie grant agreement No 753293, acronym 
NanoBEAT, and European Research Council by Starting Grant Innovative Polymers for Energy

Storage (iPes) 306250

ABBREVIATIONS

CPs, conjugated polymers; PPy, polypyrrole; PEDOT, poly(3,4-ethylenedioxythiophene);

PANi, Polyaniline; EP, electropolymerization; VPP, vapour phase polymerization; ES, electrospinning; APS, ammonium persulphate; ECM, extracellular matrix;

\section{REFERENCES}

1. $\quad$ Funk, R. H. W.; Monsees, T.; Özkucur, N., Electromagnetic effects - From cell biology to medicine. Prog. Histochem. Cytochem. 2009, 43 (4), 177-264.

2. Scanziani, M.; Häusser, M., Electrophysiology in the age of light. Nature 2009, 461, 930.

3. Clayton, R. H.; Bernus, O.; Cherry, E. M.; Dierckx, H.; Fenton, F. H.; Mirabella, L.;

Panfilov, A. V.; Sachse, F. B.; Seemann, G.; Zhang, H., Models of cardiac tissue electrophysiology: Progress, challenges and open questions. Prog. Biophys. Mol. Biol. 2011, 104 (1), 22-48.

4. Vasquez-Sancho, F.; Abdollahi, A.; Damjanovic, D.; Catalan, G., Flexoelectricity in Bones. Adv. Mater. 2018, 30 (9), 1705316.

5. $\quad$ Nezakati, T.; Seifalian, A.; Tan, A.; Seifalian, A. M., Conductive Polymers: Opportunities and Challenges in Biomedical Applications. Chem. Rev. 2018, 14, 6766-6843.

6. $\quad$ Balint, R.; Cassidy, N. J.; Cartmell, S. H., Conductive polymers: towards a smart biomaterial for tissue engineering. Acta Biomater. 2014, 10 (6), 2341-53.

7. $\quad$ Choi, J. S.; Park, J. S.; Kim, B.; Lee, B. T.; Yim, J. H., In vitro biocompatibility of vapour phase polymerised conductive scaffolds for cell lines. Polymer 2017, 124, 95-100.

8. $\quad$ Maddaly, R.; V., P.; S.R., K.; E., A.; Paul, S. F. D., 3D Cell Culture Systems: Advantages and Applications. J. Cell. Physiol. 2015, 230 (1), 16-26.

9. Jie, W.; Song, F.; Li, X.; Li, W.; Wang, R.; Jiang, Y.; Zhao, L.; Fan, Z.; Wang, J.; Liu, B., Enhancing the proliferation of MC3T3-E1 cells on casein phosphopeptide-biofunctionalized 3D reduced-graphene oxide/polypyrrole scaffolds. $R S C A d v$. 2017, 7 (55), 34415-34424.

10. Gelmi, A.; Zhang, J.; Cieslar-Pobuda, A.; Ljunngren, M. K.; Los, M. J.; Rafat, M.; Jager, E. W. H., Electroactive 3D materials for cardiac tissue engineering. In Electroactive Polymer Actuators and Devices (EAPAD) 2015, 2015.

11. Mihailescu, M.; Popescu, R. C.; Matei, A.; Acasandrei, A.; Paun, I. A.; Dinescu, M., Investigation of osteoblast cells behavior in polymeric 3D micropatterned scaffolds using digital holographic microscopy. Appl. Opt. 2014, 53 (22), 4850-8.

12. Lee, H.; Cho, Y., An Innovative Strategy for the Fabrication of Functional Cell Sheets Using an Electroactive Conducting Polymer. Theranostics 2015, 5 (9), 1021-9. 
13. Lee, J. Y.; Bashur, C. A.; Goldstein, A. S.; Schmidt, C. E., Polypyrrole-coated electrospun PLGA nanofibers for neural tissue applications. Biomaterials 2009, 30 (26), 43254335 .

14. Jin, L.; Wang, T.; Feng, Z. Q.; Zhu, M.; Leach, M. K.; Naim, Y. I.; Jiang, Q., Fabrication and characterization of a novel fluffy polypyrrole fibrous scaffold designed for 3D cell culture. $J$. Mater. Chem. 2012, 22 (35), 18321-18326.

15. Nava, M. M.; Draghi, L.; Giordano, C.; Pietrabissa, R., The Effect of Scaffold Pore Size in Cartilage Tissue Engineering. J. Appl. Biomater. Funct. Mater. 2016, 14 (3), e223-e229.

16. Tracy, R. E., Cardiomyocyte size estimated from noninvasive measurements of left ventricular wall thickness and chamber diameter. J. Am. Soc. Hypertens. 2012, 6 (3), 185-192.

17. Brown, K. M.; Gillette, T. A.; Ascoli, G. A., Quantifying neuronal size: Summing up trees and splitting the branch difference. Semin. Cell Dev. Biol. 2008, 19 (6), 485-493.

18. Severt, S. Y.; Ostrovsky-Snider, N. A.; Leger, J. M.; Murphy, A. R., Versatile Method for Producing 2D and 3D Conductive Biomaterial Composites Using Sequential Chemical and Electrochemical Polymerization. ACS Appl. Mater. Interfaces 2015, 7 (45), 25281-8.

19. Vishnoi, T.; Kumar, A., Comparative study of various delivery methods for the supply of alpha-ketoglutarate to the neural cells for tissue engineering. Biomed. Res. Int. 2013, 2013, 294679.

20. Gilmore, K.; Hodgson, A. J.; Luan, B.; Small, C. J.; Wallace, G. G., Preparation of hydrogel/conducting polymer composites. Polym. Gels Networks 1994, 2 (2), 135-143.

21. Sajesh, K. M.; Jayakumar, R.; Nair, S. V.; Chennazhi, K. P., Biocompatible conducting chitosan/polypyrrole-alginate composite scaffold for bone tissue engineering. Int. J. Biol. Macromol. 2013, 62, 465-471.

22. Wang, S.; Guan, S.; Zhu, Z.; Li, W.; Liu, T.; Ma, X., Hyaluronic acid doped-poly(3,4ethylenedioxythiophene)/chitosan/gelatin (PEDOT-HA/Cs/Gel) porous conductive scaffold for nerve regeneration. Mater. Sci. Eng. C Mater. Biol. Appl. 2017, 71, 308-316.

23. Wang, S.; Guan, S.; Xu, J.; Li, W.; Ge, D.; Sun, C.; Liu, T.; Ma, X., Neural stem cell proliferation and differentiation in the conductive PEDOT-HA/Cs/Gel scaffold for neural tissue engineering. Biomater. Sci. 2017, 5 (10), 2024-2034.

24. del Agua, I.; Marina, S.; Pitsalidis, C.; Mantione, D.; Ferro, M.; Iandolo, D.; SanchezSanchez, A.; Malliaras, G. G.; Owens, R. M.; Mecerreyes, D., Conducting Polymer Scaffolds Based on Poly(3,4-ethylenedioxythiophene) and Xanthan Gum for Live-Cell Monitoring. ACS Omega 2018, 3 (7), 7424-7431.

25. Wan, A. M.; Inal, S.; Williams, T.; Wang, K.; Leleux, P.; Estevez, L.; Giannelis, E. P.; Fischbach, C.; Malliaras, G. G.; Gourdon, D., 3D Conducting Polymer Platforms for Electrical Control of Protein Conformation and Cellular Functions. J. Mater. Chem. B 2015, 3 (25), 50405048 .

26. Zhang, J.; Li, M.; Kang, E.-T.; Neoh, K. G., Electrical stimulation of adipose-derived mesenchymal stem cells in conductive scaffolds and the roles of voltage-gated ion channels. Acta Biomater. 2016, 32, 46-56.

27. Björninen, M.; Gilmore, K.; Pelto, J.; Seppänen-Kaijansinkko, R.; Kellomäki, M.; Miettinen, S.; Wallace, G.; Grijpma, D.; Haimi, S., Electrically Stimulated Adipose Stem Cells on Polypyrrole-Coated Scaffolds for Smooth Muscle Tissue Engineering. Ann. Biomed. Eng. 2017, 45 (4), 1015-1026.

28. Giffney, T.; Xie, M.; Sartelet, M.; C Aw, K., Vapor phase polymerization of PEDOT on silicone rubber as flexible large strain sensor. AIMS Mater. Sci. 2015, 2 (4), 414-424. 
29. Iandolo, D.; Ravichandran, A.; Liu, X.; Wen, F.; Chan, J. K.; Berggren, M.; Teoh, S. H.; Simon, D. T., Development and Characterization of Organic Electronic Scaffolds for Bone Tissue Engineering. Adv. Healthc. Mater. 2016, 5 (12), 1505-12.

30. Tenhaeff, W. E.; Gleason, K. K., Initiated and Oxidative Chemical Vapor Deposition of Polymeric Thin Films: iCVD and oCVD. Adv. Funct. Mater. 2008, 18 (7), 979-992.

31. Kim, S. W.; Lee, S. W.; Kim, J.; Yim, J.-H.; Cho, K. Y., Three-dimensional, highporosity conducting skeletal structure from biodegradable microparticles with vapor-phase polymerized conformal surface layer. Polymer 2016, 102, 127-135.

32. Song, F.; Jie, W.; Zhang, T.; Li, W.; Jiang, Y.; Wan, L.; Liu, W.; Li, X.; Liu, B., Roomtemperature fabrication of a three-dimensional reduced-graphene oxide/polypyrrole/hydroxyapatite composite scaffold for bone tissue engineering. RSC Adv. 2016, 6 (95), 92804-92812.

33. Zhao, W.; Li, Y.; Wu, S.; Wang, D.; Zhao, X.; Xu, F.; Zou, M.; Zhang, H.; He, X.; Cao, A., Highly Stable Carbon Nanotube/Polyaniline Porous Network for Multifunctional Applications. ACS Appl. Mater. Interfaces 2016, 8 (49), 34027-34033.

34. Ding, Y.; Yang, J.; Tolle, C. R.; Zhu, Z., Flexible and Compressible PEDOT:PSS@Melamine Conductive Sponge Prepared via One-Step Dip Coating as Piezoresistive Pressure Sensor for Human Motion Detection. ACS Appl. Mater. Interfaces 2018, 10 (18), 16077-16086.

35. Shahini, A.; Yazdimamaghani, M.; Walker, K. J.; Eastman, M. A.; Hatami-Marbini, H.; Smith, B. J.; Ricci, J. L.; Madihally, S. V.; Vashaee, D.; Tayebi, L., 3D conductive nanocomposite scaffold for bone tissue engineering. Int. J. Nanomed. 2014, 9, 167-81.

36. Yazdimamaghani, M.; Razavi, M.; Mozafari, M.; Vashaee, D.; Kotturi, H.; Tayebi, L., Biomineralization and biocompatibility studies of bone conductive scaffolds containing poly(3,4ethylenedioxythiophene):poly(4-styrene sulfonate) (PEDOT:PSS). J. Mater. Sci. Mater. Med. 2015, 26 (12), 274.

37. Zanjanizadeh Ezazi, N.; Shahbazi, M. A.; Shatalin, Y. V.; Nadal, E.; Mäkilä, E.; Salonen, J.; Kemell, M.; Correia, A.; Hirvonen, J.; Santos, H. A., Conductive vancomycin-loaded mesoporous silica polypyrrole-based scaffolds for bone regeneration. Int. J. Pharm. 2018, 536 (1), 241-250.

38. $\quad$ Qingyong, L.; Zhengxiang, N.; Jiaoyan, R.; Wenzhen, L., Structural Design and Physicochemical Foundations of Hydrogels for Biomedical Applications. Curr. Med. Chem. 2018, 25 (8), 963-981.

39. Zhao, F.; Yao, D.; Guo, R.; Deng, L.; Dong, A.; Zhang, J., Composites of Polymer Hydrogels and Nanoparticulate Systems for Biomedical and Pharmaceutical Applications. Nanomaterials 2015, 5 (4), 2054.

40. $\quad$ Runge, M. B.; Dadsetan, M.; Baltrusaitis, J.; Ruesink, T.; Lu, L.; Windebank, A. J.; Yaszemski, M. J., Development of Electrically Conductive Oligo(polyethylene Glycol) Fumarate-Polypyrrole Hydrogels for Nerve Regeneration. Biomacromolecules 2010, 11 (11), 2845-2853.

41. Guiseppi-Elie, A., Electroconductive hydrogels: Synthesis, characterization and biomedical applications. Biomaterials 2010, 31 (10), 2701-2716.

42. Ketabat, F.; Khorshidi, S.; Karkhaneh, A., Application of minimally invasive injectable conductive hydrogels as stimulating scaffolds for myocardial tissue engineering. Polym. Int. 2018, 67 (8), 975-982. 
43. Yang, S.; Jang, L. K.; Kim, S.; Yang, J.; Yang, K.; Cho, S. W.; Lee, J. Y., Polypyrrole/Alginate Hybrid Hydrogels: Electrically Conductive and Soft Biomaterials for Human Mesenchymal Stem Cell Culture and Potential Neural Tissue Engineering Applications. Macromol. Biosci. 2016, 16 (11), 1653-1661.

44. Guo, B.; Finne-Wistrand, A.; Albertsson, A.-C., Facile Synthesis of Degradable and Electrically Conductive Polysaccharide Hydrogels. Biomacromolecules 2011, 12 (7), 2601-2609.

45. Xu, C.; Guan, S.; Wang, S.; Gong, W.; Liu, T.; Ma, X.; Sun, C., Biodegradable and electroconductive poly(3,4-ethylenedioxythiophene)/carboxymethyl chitosan hydrogels for neural tissue engineering. Mater. Sci. Eng. C Mater. Biol. Appl. 2018, 84, 32-43.

46. Mario Cheong, G. L.; Lim, K. S.; Jakubowicz, A.; Martens, P. J.; Poole-Warren, L. A.; Green, R. A., Conductive hydrogels with tailored bioactivity for implantable electrode coatings. Acta. Biomater. 2014, 10 (3), 1216-26.

47. Ravichandran, R.; Martinez, J. G.; Jager, E. W. H.; Phopase, J.; Turner, A. P. F., Type I Collagen-Derived Injectable Conductive Hydrogel Scaffolds as Glucose Sensors. ACS Appl. Mater. Interfaces 2018, 10 (19), 16244-16249.

48. Shin, J.; Choi, E. J.; Cho, J. H.; Cho, A. N.; Jin, Y.; Yang, K.; Song, C.; Cho, S. W., Three-Dimensional Electroconductive Hyaluronic Acid Hydrogels Incorporated with Carbon Nanotubes and Polypyrrole by Catechol-Mediated Dispersion Enhance Neurogenesis of Human Neural Stem Cells. Biomacromolecules 2017, 18 (10), 3060-3072.

49. Wu, Q.; Wei, J.; Xu, B.; Liu, X.; Wang, H.; Wang, W.; Wang, Q.; Liu, W., A robust, highly stretchable supramolecular polymer conductive hydrogel with self-healability and thermoprocessability. Sci. Rep. 2017, 7, 41566.

50. Mawad, D.; Artzy-Schnirman, A.; Tonkin, J.; Ramos, J.; Inal, S.; Mahat, M. M.; Darwish, N.; Zwi-Dantsis, L.; Malliaras, G. G.; Gooding, J. J.; Lauto, A.; Stevens, M. M., Electroconductive Hydrogel Based on Functional Poly(Ethylenedioxy Thiophene). Chem. Mater. 2016, 28 (17), 6080-6088.

51. Dai, T.; Jiang, X.; Hua, S.; Wang, X.; Lu, Y., Facile fabrication of conducting polymer hydrogels via supramolecular self-assembly. Chem. Commun. 2008, (36), 4279-4281.

52. Mawad, D.; Stewart, E.; Officer, D. L.; Romeo, T.; Wagner, P.; Wagner, K.; Wallace, G. G., A Single Component Conducting Polymer Hydrogel as a Scaffold for Tissue Engineering. Adv. Funct. Mater. 2012, 22 (13), 2692-2699.

53. Du, R.; Xu, Y.; Luo, Y.; Zhang, X.; Zhang, J., Synthesis of conducting polymer hydrogels with $2 \mathrm{D}$ building blocks and their potential-dependent gel-sol transitions. Chem. Commun. 2011, 47 (22), 6287-6289.

54. Holzwarth, J. M.; Ma, P. X., 3D nanofibrous scaffolds for tissue engineering. J. Mater. Chem. 2011, 21 (28), 10243-10251.

55. Tayi, A. S.; Pashuck, E. T.; Newcomb, C. J.; McClendon, M. T.; Stupp, S. I., Electrospinning Bioactive Supramolecular Polymers from Water. Biomacromolecules 2014, 15 (4), 1323-1327.

56. Niu, X.; Rouabhia, M.; Chiffot, N.; King, M. W.; Zhang, Z., An electrically conductive 3D scaffold based on a nonwoven web of poly(L-lactic acid) and conductive poly(3,4ethylenedioxythiophene). J. Biomed. Mater. Res. A 2015, 103 (8), 2635-44.

57. Chen, C.; Yu, Y.; Li, K.; Zhao, M.; Liu, L.; Yang, J.; Liu, J.; Sun, D., Facile approach to the fabrication of $3 \mathrm{D}$ electroconductive nanofibers with controlled size and conductivity templated by bacterial cellulose. Cellulose 2015, 22 (6), 3929-3939. 
58. Yow, S. Z.; Lim, T. H.; Yim, E. K. F.; Lim, C. T.; Leong, K. W., A 3D Electroactive Polypyrrole-Collagen Fibrous Scaffold for Tissue Engineering. Polymers 2011, 3 (1), 527-544.

59. Jin, L.; Feng, Z. Q.; Zhu, M. L.; Wang, T.; Leach, M. K.; Jiang, Q., A Novel Fluffy Conductive Polypyrrole Nano-Layer Coated PLLA Fibrous Scaffold for Nerve Tissue Engineering. J. Biomed. Nanotechnol. 2012, 8 (5), 779-785.

60. Pelto, J.; Bjorninen, M.; Palli, A.; Talvitie, E.; Hyttinen, J.; Mannerstrom, B.; Suuronen Seppanen, R.; Kellomaki, M.; Miettinen, S.; Haimi, S., Novel polypyrrole-coated polylactide scaffolds enhance adipose stem cell proliferation and early osteogenic differentiation. Tissue Eng., Part A 2013, 19 (7-8), 882-92.

61. Xu, Q.; Jin, L.; Li, C.; Kuddannayai, S.; Zhang, Y., The effect of electrical stimulation on cortical cells in 3D nanofibrous scaffolds. RSC Adv. 2018, 8 (20), 11027-11035.

62. Anuradha, S.; Uma Maheswari, K.; Swaminathan, S., Fabrication of uniaxially aligned 3D electrospun scaffolds for neural regeneration. Biomed. Mater. 2011, 6 (2), 025004.

63. Subramanian, A.; Krishnan, U. M.; Sethuraman, S., Axially aligned electrically conducting biodegradable nanofibers for neural regeneration. J. Mater. Sci. Mater. Med. 2012, $23(7), 1797-809$.

64. Thiéblemont, J. C.; Brun, A.; Marty, J.; Planche, M. F.; Calo, P., Thermal analysis of polypyrrole oxidation in air. Polymer 1995, 36 (8), 1605-1610.

65. Kiebooms, R.; Aleshin, A.; Hutchison, K.; Wudl, F., Thermal and Electromagnetic Behavior of Doped Poly(3,4-ethylenedioxythiophene) Films. J. Phys. Chem. B 1997, 101 (51), 11037-11039.

66. Pielichowski, K., Kinetic analysis of the thermal decomposition of polyaniline. Solid State Ionics 1997, 104 (1), 123-132.

67. Huan, Z.; Chu, H. K.; Liu, H.; Yang, J.; Sun, D., Engineered bone scaffolds with Dielectrophoresis-based patterning using 3D printing. Biomed. Microdevices 2017, 19 (4), 102. 68. Hwang Do, C.; Jung Min, H.; Tae-Yun, K.; Jin Woo, J.; Dong-Heon, H.; Dong-Woo, C., Effects of micro-patterns in three-dimensional scaffolds for tissue engineering applications. $J$. Micromech. Microeng. 2012, 22 (12), 125002.

69. Florczyk, S. J.; Wang, K.; Jana, S.; Wood, D. L.; Sytsma, S. K.; Sham, J. G.; Kievit, F. M.; Zhang, M., Porous chitosan-hyaluronic acid scaffolds as a mimic of glioblastoma microenvironment ECM. Biomaterials 2013, 34 (38), 10143-10150.

70. Jiang, L.; Gentile, C.; Lauto, A.; Cui, C.; Song, Y.; Romeo, T.; Silva, S. M.; Tang, O.; Sharma, P.; Figtree, G.; Gooding, J. J.; Mawad, D., Versatile Fabrication Approach of Conductive Hydrogels via Copolymerization with Vinyl Monomers. ACS Appl. Mater. Interfaces 2017, 9 (50), 44124-44133.

71. Spencer, A. R.; Primbetova, A.; Koppes, A. N.; Koppes, R. A.; Fenniri, H.; Annabi, N., Electroconductive Gelatin Methacryloyl-PEDOT:PSS Composite Hydrogels: Design, Synthesis, and Properties. ACS Biomaterials Science \& Engineering 2018.

72. Dewi, A. H.; Ana, I. D.; Jansen, J., Calcium carbonate hydrogel construct with cynnamaldehyde incorporated to control inflammation during surgical procedure. J. Biomed. Mater. Res., Part A 2016, 104 (3), 768-774.

73. Kwon, H.; Sun, L.; Cairns, D. M.; Rainbow, R. S.; Preda, R. C.; Kaplan, D. L.; Zeng, L., The influence of scaffold material on chondrocytes in inflammatory conditions. Acta Biomater. 2013, 9 (5), 6563-6575. 
74. Wang, S.; Sun, C.; Guan, S.; Li, W.; Xu, J.; Ge, D.; Zhuang, M.; Liu, T.; Ma, X., Chitosan/gelatin porous scaffolds assembled with conductive poly(3,4-ethylenedioxythiophene) nanoparticles for neural tissue engineering. J. Mater. Chem. B 2017, 5 (24), 4774-4788.

75. Inal, S.; Hama, A.; Ferro, M.; Pitsalidis, C.; Oziat, J.; Iandolo, D.; Pappa, A.-M.; Hadida, M.; Huerta, M.; Marchat, D.; Mailley, P.; Owens, R. M., Conducting Polymer Scaffolds for Hosting and Monitoring 3D Cell Culture. Adv. Biosyst. 2017, 1 (6).

76. Guo, W.; Zhang, X.; Yu, X.; Wang, S.; Qiu, J.; Tang, W.; Li, L.; Liu, H.; Wang, Z. L., Self-Powered Electrical Stimulation for Enhancing Neural Differentiation of Mesenchymal Stem Cells on Graphene-Poly(3,4-ethylenedioxythiophene) Hybrid Microfibers. ACS Nano 2016, 10 (5), 5086-5095.

77. Akhavan, O., Graphene scaffolds in progressive nanotechnology/stem cell-based tissue engineering of the nervous system. J. Mater. Chem. B 2016, 4 (19), 3169-3190.

78. $\quad$ Carter, M.; Shieh, J., Chapter 4 - Electrophysiology. In Guide to Research Techniques in Neuroscience (Second Edition), Carter, M.; Shieh, J., Eds. Academic Press: San Diego, 2015; pp 89-115.

79. Sayyar, S.; Bjorninen, M.; Haimi, S.; Miettinen, S.; Gilmore, K.; Grijpma, D.; Wallace, G., UV Cross-Linkable Graphene/Poly(trimethylene Carbonate) Composites for 3D Printing of Electrically Conductive Scaffolds. ACS Appl. Mater. Interfaces 2016, 8 (46), 31916-31925. 80. Kenry; Liu, B., Recent Advances in Biodegradable Conducting Polymers and Their Biomedical Applications. Biomacromolecules 2018, 19 (6), 1783-1803.

81. Reis, L. A.; Chiu, L. L.; Feric, N.; Fu, L.; Radisic, M., Biomaterials in myocardial tissue engineering. J. Tissue Eng. Regen. Med. 2016, 10 (1), 11-28.

82. Dong, R.; Zhao, X.; Guo, B.; Ma, P. X., Biocompatible Elastic Conductive Films Significantly Enhanced Myogenic Differentiation of Myoblast for Skeletal Muscle Regeneration. Biomacromolecules 2017, 18 (9), 2808-2819.

83. Lee, J.-W.; Serna, F.; Nickels, J.; Schmidt, C. E., Carboxylic Acid-Functionalized Conductive Polypyrrole as a Bioactive Platform for Cell Adhesion. Biomacromolecules 2006, 7 (6), 1692-1695.

84. Yow, S.-Z.; Lim, T. H.; Yim, E. K. F.; Lim, C. T.; Leong, K. W., A 3D Electroactive Polypyrrole-Collagen Fibrous Scaffold for Tissue Engineering. Polymers 2011, 3 (1), 527-544.

85. Balint, R.; Cassidy, N. J.; Cartmell, S. H., Electrical Stimulation: A Novel Tool for Tissue Engineering. Tissue Eng., Part B 2012, 19 (1), 48-57.

86. Daish, C.; Blanchard, R.; Fox, K.; Pivonka, P.; Pirogova, E., The Application of Pulsed Electromagnetic Fields (PEMFs) for Bone Fracture Repair: Past and Perspective Findings. Ann. Biomed. Eng. 2018, 46 (4), 525-542.

87. Instruments, W. P. https://www.wpiinc.com/electrophysiology/electrodes.

88. Chikar, J. A.; Hendricks, J. L.; Richardson-Burns, S. M.; Raphael, Y.; Pfingst, B. E.; Martin, D. C., The use of a dual PEDOT and RGD-functionalized alginate hydrogel coating to provide sustained drug delivery and improved cochlear implant function. Biomaterials 2012, 33 (7), 1982-90.

89. Cysewska, K.; Karczewski, J.; Jasiński, P., Recurrent potential pulse technique for improvement of glucose sensing ability of 3D polypyrrole. Meas. Sci. Technol. 2017, 28 (7). 90. Ligon, S. C.; Liska, R.; Stampfl, J.; Gurr, M.; Mülhaupt, R., Polymers for 3D Printing and Customized Additive Manufacturing. Chem. Rev. 2017, 117 (15), 10212-10290.

91. Mu, X.; Bertron, T.; Dunn, C.; Qiao, H.; Wu, J.; Zhao, Z.; Saldana, C.; Qi, H. J., Porous polymeric materials by 3D printing of photocurable resin. Mater. Horiz. 2017, 4 (3), 442-449. 
92. Dong, S. L.; Han, L.; Du, C. X.; Wang, X. Y.; Li, L. H.; Wei, Y., 3D Printing of Aniline Tetramer-Grafted-Polyethylenimine and Pluronic F127 Composites for Electroactive Scaffolds. Macromol. Rapid Commun. 2017, 38 (4). 